BULLETIN Bulletin hispanique

HISPANIQUE Université Michel de Montaigne Bordeaux

117-2 | 2015

Métamorphose(s) : représentations et réécritures

\title{
La métamorphose marine de Cola Pesce
}

mythe, folklore, littérature

\section{François Delpech}

\section{(2) OpenEdition}

Journals

Édition électronique

URL : http://journals.openedition.org/bulletinhispanique/4091

DOI : 10.4000/bulletinhispanique.4091

ISSN : 1775-3821

Éditeur

Presses universitaires de Bordeaux

Édition imprimée

Date de publication : 15 décembre 2015

Pagination : 615-644

ISBN : 979-10-300-0041-2

ISSN : 0007-4640

Référence électronique

François Delpech, « La métamorphose marine de Cola Pesce », Bulletin hispanique [En ligne],

117-2 | 2015, mis en ligne le 15 décembre 2018, consulté le 05 mai 2019. URL : http://

journals.openedition.org/bulletinhispanique/4091; DOI : 10.4000/bulletinhispanique.4091 


\title{
La métamorphose marine de Cola Pesce : mythe, folklore, littérature
}

\author{
François Delpech \\ CRES Paris III et UMR 7192 - CNRS - Collège de France
}

Ovide évoque des transformations d'êtres humains en poissons ou en créatures hybrides ichtyomorphes, parfois divines. Ces récits sont recyclés, du Moyen Âge à l'Époque Moderne, dans les cultures folklorique et littéraire d'Italie et d'Espagne, à propos de l'Homme Marin de Messine ("Cola Pesce »). On discerne dans ce corpus ce qui relève de la tradition poético-mythographique, et ce qui reconduit aux survivances locales d'une archaïque mythologie méditerranéenne.

Mots-clés : Ovide, métamorphoses, Cola Pesce, l'Homme Marin.

Ovidio alude a transformaciones de seres humanos en peces o en criaturas híbridas ictiomorfas, a veces divinas. En el Medioevo y en la época moderna estos relatos se reciclan en las culturas folklórica y literaria de Italia y España a propósito del Hombre Marino de Mesina ("Cola Pesce»). Se examina lo que remite, en estas ficciones, a la tradición poético-mitográfica y lo que procede de supervivencias locales de una arcaica mitología mediterránea.

Palabras claves: Ovidio, metamorfosis, Cola Pesce, Hombre marino.

Ovid mentions the transformations of human beings into fishes, or ichthyomorphous hybrid creatures, sometimes said to be divinities. Since the Middle Ages, these tales have been recycled, in Italy and Spain, through literary and oral traditions about the Merman of Messina ("Cola Pesce»). These legends are analysed both as part of a learned poetic and mythographic culture, as well as local survivals of an archaic mediterranean mythology.

Keywords: Ovid, Metamorphoses, Cola Pesce, Merman. 
$\mathrm{O}$ N SAIt, Depuis les publications savantes de Giuseppe Pitrè et de Benedetto Croce ${ }^{1}$, aujourd'hui complétées par des enquêtes beaucoup plus récentes ${ }^{2}$, quelle a été l'étonnante fortune de ce fleuron du légendaire et de l'imaginaire méditerranéens que fut le personnage de "Cola Pesce».

Ce prodigieux nageur du détroit de Messine, qu'une malédiction maternelle avait condamné à se transformer en une sorte d'homme marin, et qu'une abusive injonction royale avait conduit à trouver la mort au sein du gouffre (où le souverain avait jeté une coupe d'or en lui demandant de la rapporter), a fait l'objet, du XII siècle à nos jours, notamment en Sicile, à Naples, en Espagne et même au-delà, d'une double élaboration légendaire.

C'est d'abord - dans le registre de la " culture savante " - un abondant corpus de textes littéraires relevant des genres les plus divers (miscellanées érudites, poésie, théâtre $)^{3}$, où l'on relève, entre autres noms prestigieux, ceux de Pontano, Pedro Mexía, Cervantès, Schiller, L. Sciascia, etc.

Mais c'est aussi - dans le domaine des traditions orales - un bon nombre de versions "folkloriques " dialectales, notamment sous forme de légendes topographiques et d'énoncés de croyances (surtout en Sicile, dans la région de Messine, mais aussi à Naples, en Andalousie, en Catalogne, et jusqu'en Bretagne $)^{4}$, constitutives d'un corpus parallèle, probablement plus ancien que celui de la tradition écrite, qui semble en être tributaire et entretient avec lui des relations apparemment complexes.

Ayant examiné, dans un autre contexte, quelques-unes des probables racines mythiques et la principale connexion hagiographique (avec la légende

1. Giuseppe Pitrè, Studi di leggende popolari in Sicilia e nuova raccolta di leggende siciliane, Turin, C. Clausen, 1904, p. 1-173, Benedetto Croce, Storie e leggende napoletane (rééd.), Milan, Adelphi, 1990, p. 298-305.

2. Anita Seppilli, Sacralità dell'acqua e sacrilegio dei ponti, Palerme, Sellerio, $1990^{2}$, p. 294348, Giuseppe Cavarra, La leggenda di Colapesce, Messine, Intilla, 1998 ; Maria D’Agostino, La leggenda di Cola Pesce. Una versione spagnola del secolo XVII, Rome, Salerno, 2008 ; Giovanni Battista Bronzini, "Cola Pesce e il tuffatore. Dalla leggenda moderna al mito antico ", Lares, 66, 2000, p. 341-376 ; Fiorella La Guardia, «La leggenda di Cola Pesce, fra mito antico e studi moderni », Lares, 69 (3), 2003, p. 535-562 ; Consiglia Landi, « Niccolò Pesce. Un monumento napoletano ed una leggenda ", Quaderni dell'Accademia Pontaniana, 2, 1981, p. 30-80. On trouvera dans les études de G. B. Bronzini, op. cit., et M. D’Agostino, op. cit., les références des multiples articles de B. Croce consacrés à cette légende.

3. La plupart de ces textes sont reproduits in G. Pitrè, op. cit., G. Cavarra, op. cit., et M. D’Agostino, op. cit.

4. Cf. les versions recueillies en Sicile par G. Pitrè, op. cit., p. 150-173, et G. Cavarra, op. cit., p. 109-154. Pour leur mise en parallèle avec les versions littéraires cf. F. La Guardia, op. cit, p. 550-558. Pour une version napolitaine cf. B. Croce, op. cit., loc. cit.. Une version catalane a été recueillie par Joan Amades, Folklore de Catalunya. Rondallistica, Barcelone, Selecta, 1950, p. 1138-1139, n 1604, «El peix Nicolau ». Pour les versions bretonnes cf. Paul Sébillot, Folklore de France, Paris, Maisonneuve et Larose, 1968², tome II, p. 134-136 (lutins protéiformes nommés " Nicole ", ou "Collé "; bête marine agressive appelée "Saint-Nicolas »), et Pierre J. Hélias, Légendes de la mer, Chateaulin, Jos Le Douaré, 1958, p. 89-91, "Le joyeux lutin Nicole ». Sur la diffusion de la légende en Espagne cf. Julio Caro Baroja, "El "Pesce Cola" o "El Peje Nicolao" ", Revista de Dialectología y Tradiciones Populares, 39, 1984, p. 7-16, et M. D’Agostino, op. cit. 
de saint Nicolas) de ce double cycle traditionnel ${ }^{5}$, je m'intéresserai ici plus particulièrement au thème de la métamorphose marine, qui en constitue l'un des pivots essentiels.

\section{TRADITION LITTÉRAIRE, TRADITION FOLKLORIQUE}

Le thème en question apparait aussi bien dans les versions littéraires de notre légende, quoique les plus anciennes ne le mentionnent $\mathrm{pas}^{6}$, que dans les versions folkloriques, qui lui donnent généralement plus d'ampleur et de relief : ces dernières accentuent en effet l'aspect fabuleux du personnage, la dimension quasi "mythologique " de sa geste, alors que les textes de culture écrite tendent plutôt, du moins dans certains cas, à présenter Cola Pesce comme un personnage "réel ", un nageur exceptionnel à qui ses dons nautiques et son destin fatal auraient conféré une aura légendaire d'abord régionale puis nationale et internationale.

C'est donc à un double titre qu'un paramètre " ovidien " peut être éventuellement subodoré dans les élaborations diverses de notre légende sicilienne.

1/ - Relevant d'une culture écrite hypersavante, la tradition du livre des Métamorphoses et de ses interprètes du Moyen Âge et de la Renaissance était nécessairement présente à l'esprit des érudits et des lettrés qui ont eu à traiter l'histoire du fabuleux nageur de Messine et de son détroit, région éminemment chargée de réminiscences mythographiques (celles concernant Charybde et Scylla étant les plus fameuses, et pour ainsi dire proverbiales). Dans le long carmen néolatin consacré par Giovanni Pontano à Cola Pesce dans le quatrième livre de son Urania sive de stellis, le poète humaniste ne manque pas, par exemple, d'évoquer le combat de son héros avec Scylla, qu'il met en fuite après l'avoir blessée d'un coup de glaive ${ }^{7}$, mais ce sera pour périr ensuite sous les coups de Charybde, qui le précipite et le déchire sur les écueils ${ }^{8}$. Dans cette ébauche

5. F. Delpech, « De saint Nicolas à Cola Pesce : mythe, hagiographie et folklore autour de la légende du prodigieux nageur de Sicile ", paru dans Memorie del Mare. Divinità, santi, eroi, navigatori (Atti del Convegno Internazionale, Cefalù 4-6 novembre 2010), S. Mannia (éd.), Palerme: Fondazione Ignazio Buttitta, 2011, p. 129-169.

6. Les premières sont celles de Walter Map et Raimon Jordan (XII siècle) : cf. M. D’Agostino, op. cit., p. 108-111, où elles sont reproduites. Ce n'est qu'à partir de la fin du XIII' siècle (texte de la chronique de Salimbene de Adam : cf. ibid., p. 110-111) qu'apparaît une ébauche de biographie du personnage. Les premières allusions à une " métamorphose " physique de Nicolas (pourtant déjà appelé "Piscis ", dès le Chronicon de F. Pipino da Bologna, XIVe siècle : ibid., p. 112-113) figurent dans le De Immanitate de G. Pontano et les Commentaires de R. Maffei da Volterra, c'est-à-dire au XV' siècle (ibid., p. 120-122 et 122-123) : elles semblent cependant n'affecter que l'aspect extérieur (" effigiem ", «tanquam marinum monstrum»), non la physiologie ni la nature intrinsèque du personnage.

7. M. D'Agostino, op. cit., p. 114-121. Il n'y a pas d'équivalent ovidien de ce genre de scène concernant Scylla.

8. Ibid., p. 120-121. 
d'épopée chevaleresque sous-marine, à laquelle font écho les premières suites du Lazarillo (où le jeune pícaro, transformé en thon, participe à une guerre subaquatique $)^{9}$, il n'est cependant pas fait allusion à une " métamorphose " physique de Cola (dont l'onomastique ne comporte pas en l'occurrence de surnom ichthyologique).

Il n'en va pas de même, par contre, dans un autre texte latin de Pontano - cette fois en prose - sur la même légende, où les références mythographiques font défaut, mais où, en revanche, le thème central du poème d'Ovide - celui du changement de nature - passe au premier plan : Cola y retrouve son surnom ( "Piscis») et surtout il perd son humanité et se voit transformé en bête marine " ut non hominis mores tantum exuerit, verum etiam ipsam pene effigiem, lividus, squamosus, horridus $»^{10}$. Cette métamorphose n'a cependant rien de surnaturel ou de divin : elle est le résultat progressif d'un changement de biosphère et d'une désocialisation ("...relicta humana societate omnem fere vitam ab ipsa pueritia in mari egit atque inter pisces...").

Tout se passe donc comme si l'auteur napolitain, qui se voulait à la fois poète et homme de science, avait, à propos de la même légende, séparément recyclé deux aspects distincts de l'héritage ovidien sans jamais réussir à en faire la synthèse : d'une part il en réactive - à coups de références littéraires l'imagerie poétique, sans entreprendre pour autant de redonner vie au mythe métamorphique qui la sous-tendait ; d'autre part il tente de rationaliser et moraliser le thème du changement de nature et d'aspect, ce qui l'amène à ignorer la dimension mystérique de la grandiose théologie païenne qui traversait de part en part le poème ovidien.

2/ - C'est par contre, paradoxalement, vers les versions folkloriques de notre légende sicilienne (et vers ceux des textes littéraires qui en portent plus fidèlement et particulièrement la marque) qu'il faut se tourner pour retrouver quelque chose de cette inspiration antique et mythique. C'est dans ces traditions, probablement élaborées et manifestement véhiculées par des illettrés, pêcheurs et marins des côtes de la Méditerranée occidentale, qui ne savaient rien d'Ovide ni de la culture des écoliers et des humanistes, que l'on décèle les équivalents les plus visibles du système d'images et de représentations dont le poète des Métamorphoses et ses sources ont été directement ou indirectement tributaires.

9. Cf. F. Delpech, "Lazare, l'eau, le vin et les thons. Éléments pour une recherche sur les corrélats folkloriques de la première continuation du Lazarillo (Anvers, 1555) " in Ecritures, pouvoirs et société en Espagne aux XVIe et XVII' siècles (Hommage du CRES à Augustin Redondo), Paris, Publ. de la Sorbonne - Presses de la Sorbonne Nouvelle, 2001, p. 309-327.

10. Ioannis Ioviani Pontani, De immanitate liber, L. Monti Sabia (éd.), Naples, Loffredo, 1970, chap. I, p. 5-6. Sur l'influence d'Ovide sur Pontano et sur son appropriation du thème de la métamorphose, cf. Francesco Tateo, "Ovidio nell' Urania di Pontano ", in I. Gallo et L. Nicastri (éds.), "Aetates Ovidianae ». Lettori di Ovidio dall' Antichità al Rinascimento, Naples, Ediz. Scient. Ital., 1995, p. 279-291, et Donatella Coppini, "Le metamorfosi del Pontano ", in G. M. Anselmi et M. Guerra (éds.), Le Metamorfosi di Ovidio nella letteratura tra Medioevo e Rinascimento, Bologne, Gedit, 2006, p. 75-108 (en particulier p. 84-87). 
Pour mieux saisir les clivages entre les versions orales et les réécritures lettrées de la légende sicilienne et tenter de repérer leurs occasionnels et implicites entrelacements, opérations préalables à toute tentative de mise en perspective de ce double corpus vis-à-vis de la tradition ovidienne, voyons ce qu'il en est de la nature et du statut ontologique du personnage en question et en quoi consiste(nt), selon les versions, la (ou les) métamorphose(s) qui l'affecte(nt).

Notons d'abord que son onomastique duelle est le plus souvent inexacte, voire équivoque. "Cola Pesce " (ou "Piscicola", "El pez Nicolao ") n'a fréquemment de poissons que le (sur)nom ${ }^{11}$. C'est en effet, dans la plupart des cas, un personnage mixte, doté de caractéristiques physiologiques plus ou moins secondaires - affectant notamment son système respiratoire (qui lui permet de rester indéfiniment sous l'eau) - qui le rendent compatible avec le milieu marin.

Généralement les versions littéraires s'en tiennent là : sous-entendant que ces caractéristiques procèdent d'un processus naturel d'adaptation, elles indiquent qu'il s'agit bien, fondamentalement, d'un homme ${ }^{12}$, qu'il lui arrive de parler et d'entretenir d'occasionnels rapports avec d'autres hommes, comme c'est le cas lorsqu'il obéit au roi qui lui commande d'aller chercher la coupe jetée dans l'abysse, ou lorsqu'il renseigne les marins sur les courants et les périls du détroit. Dans ces cas son anormalité tient plus à ses performances "sportives " exceptionnelles (de nageur et plongeur émérite) qu’à sa nature.

Les versions folkloriques développent par contre les traits aquatiques et/ou ichthyomorphiques du personnage, le dotant souvent de doigts à membranes et parfois d'écailles. Elles évoquent dans certains cas une véritable hybridité, la partie inférieure du corps apparaissant, selon le type sirénien, analogue à la queue d'un poisson ${ }^{13}$. Seules certaines versions périphériques, notamment bretonnes, en font un poisson complet, dépourvu de tout anthropomorphisme ${ }^{14}$. Dans quelques versions, qui ont eu apparemment un enracinement privilégié dans la péninsule ibérique, l'incompatibilité avec le milieu terrestre est telle que Cola ne peut quitter la mer sans mourir et que, lorsqu'il doit venir à terre (par exemple pour assister au mariage de sa sœur !), il est obligé de s’y faire transporter dans un tonneau plein d'eau ${ }^{15} \ldots$

11. Sur la figure de «l'homme-poisson » dans les fêtes, rituels et représentations dramatiques populaires en Italie et en Sicile, cf. Carmelina Naselli, «Luomo-pesce nella novellistica e nelle rappresentazioni sceniche popolari ", in Etnografia e folklore del mare, Naples, 1957, p. 543-550, et Sergio Bonanziga et Marta Di Mariano, « Una pesce mimata per incantare il mare. Il dramma dell'uomo-pesce nella Riviera dei Ciclopi ", in I. E. Buttitta et M. E. Palmisano (éds.), Santi a mare. Ritualità e devozione nelle comunità costiere siciliane, Palerme, Regione Siciliana, 2009, p. 67-85, où sont discutées les relations éventuelles de la légende de Cola Pesce avec cette figure rituelle.

12. La version de W. Map indique que Nicolas n'a " nichil inhumanum in membris" (M. D’Agostino, op. cit., p. 108).

13. Beaucoup de versions orales précisent que Cola est "menzu omu e menzu pisci».

14. Cf. P. J. Hélias, op. cit., le "lutin Nicole " est un pêcheur qui a été métamorphosé en marsouin car il était allé pêcher un dimanche.

15. Cf. la version catalane recueillie par J. Amades, op. cit., et le romance espagnol dont il sera 
On pourrait donc être tenté de ne voir en Cola Pesce qu'une variante parmi d'autres d'un type déjà bien implanté dans l'imaginaire médiéval européen, celui de l' "Homme Marin ", représentant d'une espèce instable et mal définie, plus ou moins intermédiaire entre l'humanité et l'animalité, généralement hybride et partiellement amphibie, considérée comme existant réellement au sein des mers et des océans. Equivalent masculin de la sirène, empruntant ses caractéristiques d'une part à la mythographie et à l'iconographie antiques (les «tritons » et autres "Vieux de la mer ") $)^{16}$, d'autre part aux traditions topographiques propres à la culture folklorique des régions côtières (notamment celtiques) relatives aux "génies de la mer », ce personnage topique et composite relève à la fois de la cosmographie savante, des récits de navigation, de la littérature d'imagination, de la poésie et des légendes orales des pêcheurs ${ }^{17}$. Sa polyvalence statutaire et sa labilité formelle lui permettent de circuler simultanément sur plusieurs registres culturels avec d'autant plus de facilité que " culture savante » et " culture populaire » ont longtemps partagé l'idée qu'il doit y avoir une sorte de symétrie spéculaire - plus ou moins inversée selon les cas - entre monde marin et monde terrestre, une réplique de l'humanité terrienne devant nécessairement exister, avec les adaptations nécessaires, au sein des abysses ${ }^{18}$.

question plus loin. Le nom de "Pipe » ou "Pipa ", ajouté à celui de Nicolas dans les versions médiévales de W. Map et G. de Tilbury, fait peut-être indirectement allusion à ce motif (noter que le romance appelle "pipa» le récipient en question).

16. Sur ce type de personnage mythique dans les traditions indo-européennes cf. Claude Sterckx, Les dieux protéens des Celtes et des Indo-Européens, Mémoires de la Société Belge d'Études Celtiques, n 4, Bruxelles, S.B.E.C., 1994, et Dominique Briquel, "Vieux de la mer grecs et Descendant des eaux indo-européen ", in R. Bloch (éd.), D'Héraclès à Poséidon. Mythologie et protohistoire, Genève-Paris, Droz-Champion, 1985, p. 141-158.

17. Cf. Klaus Joachim Heinisch, Der Wassermensch. Entwicklungsgeschichte eines Sagenmotivs, Stuttgart, Klett-Cotta, 1981, Beate Otto, Unterwasser-Literatur. Von Wasserfrauen und Wassermännern, Wurzburg, Königshausen-Neumann, 2001², Claude Lecouteux, "Les génies des eaux : un aperçu ", in D. James-Raoul et C. Thomasset (éds.), Dans l'Eau, sous l'Eau. Le monde aquatique au Moyen Age, Presses de l'Univ. de Paris-Sorbonne, 2002, p. 253-270 (cf., dans le même volume, les articles de I. Vedrenne, «L'homme sous la mer. La figure du plongeur dans le monde gréco-romain et l'Occident médiéval », p. 273-319, et de H. Toelle, «Des pêcheurs de perles aux Ginn sous-marins », p. 321-337). Cf. également C. Sterckx, op. cit., et, pour le domaine ibérique, J. Caro Baroja, "La creencia en hombres marinos », in Algunos mitos españoles, Madrid, Ed. del Centro, $1974^{3}$, p. 133-144, et Xoán Xosé Teijeiro Rey, Seres galegos das augas. Mitoloxía comparada, La Corogne, Toxosoutos, 2002. Sur les formes médiévales de cette mythologie cf. Mia I. Gerhardt, Old Men of the Sea. From Neptunus to Old French "luiton" : ancestry and character of a water-spirit, Amsterdam, Polak - Van Gennep, 1967, et P. Sébillot, op. cit.

18. Cf. Jacqueline Leclercq-Marx, "L'idée d'un monde marin parallèle au monde terrestre : émergence et développements ", in C. Connochie-Bourgne (éd.), Mondes marins du Moyen Age, Senefiance, 52, 2006, p. 259-271. Les versions de notre légende présentées par G. de Tilbury et P. Bersuire font allusion à des forêts sous-marines (M. D'Agostino, op. cit., p. 108-109 et 114115). Ce genre de récits remonte au moins aux versions du Roman d'Alexandre où figure l'épisode de l'exploration sous-marine du héros macédonien. Cf. aussi, dans les 1001 Nuits, le conte "Abdallah de la terre et Abdallah de la mer ". 
Cola Pesce a certes des caractéristiques communes avec cet archétype, mais il s'en distingue nettement par un faisceau de singularités qui ont permis d'individualiser et de fixer son image dans la mémoire collective. Au nombre de ses notes spécifiques on retiendra le fait qu'il n'est pas le représentant d'une espèce vivante, le membre anonyme d'une collectivité d'êtres marins indifférenciés (comme les tritons ou les sirènes) : c'est un individu sui generis, un cas unique et porteur, en tant que tel, d'un nom propre. D'autre part, malgré ses traits plus ou moins hybrides, l'humanité reste en lui dominante, comme l'attestent son aptitude au langage et les liens fugitifs qu'il entretient avec le pouvoir et avec d'autres hommes. Enfin et surtout, engendré par des parents humains et terriens (apuliens ou siciliens selon les versions), ce n'est nullement un homme marin de naissance : à la différence de ces créatures maritimes hybrides issues par exemple des amours d'un pêcheur et d'une sirène ou du rapt ripuaire d'une fille de la côte par quelque triton en goguette occasionnellement sorti de son élément ${ }^{19}$, Cola est né normalement et a eu une enfance terrienne ordinaire, seulement marquée par une vive inclination, d'origine inconnue, pour les ébats nautiques. Jusqu'au jour où, suite à un événement sur lequel on reviendra plus loin, sa vie a irréversiblement basculé, scindée en un avant et un après. C'est une nouvelle existence, entièrement marine, qui commence brusquement pour lui dès le moment où il plonge dans le détroit, pour ne plus reparaître que de temps en temps, jusqu'à l'épisode tragique de la «noyade » - ou du moins de l’immersion définitive - déterminée par le caprice royal.

Contrairement aux " hommes marins" traditionnels, dont la vie semble continue et monotone, Cola Pesce peut donc prétendre à une sorte de statut héroïque car il est doté d'une biographie. Biographie personnelle, duelle et accidentée, singularisée par un changement soudain de milieu, de nature et de statut : sa condition et sa constitution physique et morale d'habitant des mers ont été acquises à un moment déterminé, en rupture quasi complète avec sa vie antérieure, sans que soit clairement expliqué si ses modifications physiologiques ont été instantanées ou progressives (voire invisiblement inscrites depuis toujours dans ses gènes, comme pourraient le laisser supposer ses prédispositions enfantines pour les jeux et sports aquatiques).

Toujours est-il qu'il a subi une transformation, corrélative de la première irréversible immersion, et que son destin exceptionnel et solitaire s'est scellé lorsqu'il s'est ainsi retrouvé presque complètement coupé de la communauté humaine, sans pour autant se voir intégré dans une quelconque collectivité marine.

Dès lors son statut apparait éminemment ambivalent : les occasionnels services qu'il rend à des navigateurs et l'excessive dépendance qu'il manifeste à

19. Cf. F. Delpech, "La fable de l'hybride : Antonio de Torquemada et l'Homme Marin ", in N. Peyrebonne et P. Renoux-Caron (éds.), Le milieu naturel en Espagne et en Italie. Savoirs et représentations, $X V^{E}-X V I I^{e}$ s., Paris, Presses de la Sorbonne Nouvelle, 2011, p. 75-101, et Marcel Turbiaux, "L'homme sauvage aquatique ", Bulletin de la Société de Mythologie Française, 209, 2002, p. 9-19. 
l'égard du roi abusif traduisent à la fois une option sociabilisante et la sujétion suicidaire à un ordre politique vécu comme un fatum; quant à l'apparente liberté que devraient lui garantir ses perpétuels déplacements de par les espaces amers, elle ne semble être que l'insuffisante contrepartie d'une vie vouée à l'exclusion solitaire. Tout se passe comme si Cola Pesce était pris dans un « rite de passage " inabouti et se trouvait ainsi maintenu de façon permanente dans un stade de marge qui aurait dû normalement n'être que transitoire ${ }^{20}$.

On voit donc en quoi la légende du prodigieux nageur de Messine peut, dans une certaine mesure, être considérée comme une variation spécifique (et partiellement aberrante) sur le thème qui sous-tend le poème ovidien et sa postérité. La nature et le modus vivendi de Cola Pesce résultent bien d'une métamorphose, relativement analogue à celles qui sont décrites dans les récits de la tradition mythographique. Analogie dont je soupçonne qu'elle ne résulte pas d'un emprunt ou d'une influence (si ce n'est dans les marges les plus " littéraires " de notre corpus), mais plutôt d'un enracinement commun dans d'anciennes légendes étiologiques et topographiques, transmises oralement, où cette littérature gréco-latine savante semble avoir puisé l'essentiel de sa matière.

\section{MÉTAMORPHOSES ET APOTHÉOSES}

Comme beaucoup de métamorphoses ovidiennes, celle de Cola Pesce suppose un changement profond de statut, sinon une altération radicale de la nature humaine du personnage, assorti d'une modification plus ou moins complète de son apparence physique. Cette transformation s'est opérée en une seule fois, d'une manière quasi instantanée, et elle est irréversible. Elle résulte, au moins implicitement, d'une situation antérieure qui l'a rendue inévitable et potentiellement prévisible, comme on peut en juger a priori par les étranges pulsions hydrophiles de l'enfant, causes de l'irritation croissante des parents, et a posteriori par le tour fatal, comme mystérieusement surdéterminé, que prendront après coup les événements. Certes cette métamorphose n'est pas d'origine divine ni l'œuvre d'un magicien vindicatif, mais elle résulte bien du courroux d'une entité ayant autorité sur le protagoniste (la mère en colère, dont la parole de malédiction semble soudainement et surnaturellement revêtue d'un pouvoir aussi inexplicablement contraignant que le sera l'ordre lancé par le roi, auquel Cola obéira passivement tout en sachant qu'il en mourra). Enfin, comme on le verra plus loin, la métamorphose, opérant comme un rite initiatique, confere, au moins dans certaines versions de notre légende, une dimension suprahumaine au personnage qui en est l'objet et l'institue comme entité quasi mythologique.

20. La légende sicilienne se distingue donc bien du mythe classique relatif au plongeon et à l'investiture sous-marine de Thésée, auquel on l'a souvent comparée, lequel porte le processus " initiatique " à son terme et implique in fine un retour définitif du héros à la vie "terrienne " (Bacchylides XVII). 
Dans les textes antiques cette extrapolation ontologique, punitive ou glorifiante, qui peut aussi bien apparaître comme apothéose divinisatrice, ou projection dans les astres, que comme chute dans la matière inerte, le bestial ou le monstrueux, apparaît de même souvent ambivalente : les pirates transformés en dauphins par Dionysos pour les punir de leur projet sacrilège perdent leur humanité et deviennent des bêtes (comme Actéon), mais cette métamorphose les propulse à leur corps défendant dans la sphère du divin, ces mêmes dauphins étant voués à devenir in fine membres du thiase maritime dionysiaque ${ }^{21}$. Une analogue ambivalence affecte le destin de Cola Pesce : maudit par sa mère pour sa désobéissance, il subit une transformation en créature semi bestiale, puis en sur-obéissant à l'ordre abusif de l'entité paternelle que représente le roi il accède virtuellement, par sa disparition sans retour dans les profondeurs de l'au-delà subaquatique, au statut intemporel de génie tutélaire des espaces marins.

Si l'on examine maintenant plus particulièrement les histoires ovidiennes de métamorphoses d'humains en créatures de la mer, on constate que, œuvres d'un dieu ou d'une magicienne, elles sont comme il se doit d'origine et de caractère surnaturel. Outre le cas, cité plus haut, des pirates tyrrhéniens transformés en dauphins par Dionysos, on connaît la métamorphose de Scylla en monstre marin par $\mathrm{Circé}^{22}$, ou celle des amants de la naïade indienne, fille du Soleil, devenus poissons sous l'effet des incantations et des filtres de cette dernière (qui en sera punie par son père en subissant à son tour le même sort, tandis que, redevenues humaines, ses victimes seront à l'origine du peuple étrange des Ichthyophages) ${ }^{23}$. Quant à Dercetis et Vénus qui, quoique citées séparément, représentent une même divinité, équivalent hellénisé de la « Déesse syrienne » ${ }^{24}$, on sait qu'elles se jettent à l'eau et se transforment en poissons pour échapper à un sort funeste, donnant ainsi naissance à un culte ichthyolâtre.

Qu'elles sanctionnent une transgression, agencent un salut miraculeux ou résultent d'une intention hostile, qu'elles soient volontaires et autoinfligées ou subies, ces métamorphoses - qui sont dans la plupart des cas des ichthyomorphoses ${ }^{25}$ - correspondent à une transformation complète qui fait disparaitre toute apparence humaine (même Scylla, d'abord transformée en monstre dans la seule partie inférieure de son corps, devient finalement et intégralement rocher) et relèvent du merveilleux, voire de la thaumatologie maléfique $^{26}$. Ceux qui pâtissent ou bénéficient de ces métamorphoses, ou

21. Ovide, Mét., III, 582-691. Cf. Pierre Somville, «Le dauphin dans la Religion Grecque ", Revue de l'Histoire des Religions", 201 (1), 1984, p. 3-24 (p. 14-24).

22. Ovide, Mét., XIII, 898-968, XIV, 1-74.

23. Ibid., IV, 49-51. C'est précisément à propos des Ichthyophages que Pierre Bersuire introduit (en s'inspirant du texte de Gervais de Tilbury) la légende de «Nicolaus Piscis» dans son Reductorium morale (M. D’Agostino, op. cit., p. 114-115).

24. Cf. Paul Perdrizet, "Légendes babyloniennes dans les Métamorphoses d'Ovide ", Revue de l'Histoire des Religions, 105, 1932, p. 193-238. Cf. infra nn. 98-100.

25. Cf. le cas de "Pompilos ", transformé en poisson-pilote par Apollon pour avoir aidé Ocyrrhoé à lui échapper, cité par Élien et Apollodore (cf. Paul M. C. Forbes Irving, Metamorphosis in Greek Myth, Oxford, Clarandon Pr., 1990, p. 318).

26. Cf. le cas des Telkhines (Ovide, Mét., VII, 365-366) sur lesquels voir Bernard Sergent, Le 
parfois - comme Protée - ont le pouvoir de se métamorphoser eux-mêmes à volonté, appartiennent directement ou indirectement à la sphère du sacré (fût-ce d'un "sacré de transgression ") ou en portent la marque indélébile. Enfin leurs transformations apparaissent comme des métaphores objectivées de l'élément marin lui-même, lié plus que tout autre au mouvement, à l'instabilité, à l'insaisissable. Aussi sont-elles souvent étroitement corrélées à une immersion, et lui sont-elles généralement consécutives, lorsque toutefois les changements de forme et de milieu ne se produisent pas simultanément.

S'il est vrai que Cola Pesce se distingue de ces personnages mythologiques en ce qu'il ne subit qu'une métamorphose partielle (en cela il ressemble aux Telkhines ${ }^{27}$, présentée plus ou moins confusément dans certains textes comme relevant d'un phénomène naturel d'adaptation au milieu marin, sans qu'aucune allusion explicite soit faite à une intervention divine ni à un rituel magique, s'il est également patent qu'il ne devient ni un animal (sauf versions rares et périphériques), ni une divinité ou un immortel génie des eaux, ni à proprement parler un monstre, son dossier légendaire présente néanmoins de curieuses affinités, plus ou moins marquées selon les versions, avec le cycle mythologique des métamorphoses marines, notamment avec quelques-unes de celles qui ont été retenues et particulièrement développées par Ovide.

Avant d'examiner plus précisément quelques-unes de ces affinités, on remarquera qu'elles ne sont probablement ni fortuites ni simplement dues à des réminiscences littéraires transfusées dans la tradition orale. Plusieurs indices permettent en effet de supposer que cette dernière n' est autre que la continuation transposée et recyclée d'une mythologie locale sous-jacente remontant au moins à la fin de l'Antiquité, moyennant notamment sa contamination avec le cycle hagiographique de saint Nicolas, personnage lui-même syncrétique et complexe, non exempt de composantes "païennes ${ }^{28}$.

Quelques versions, exclusivement orales et siciliennes, de la légende font en effet de Cola Pesce une sorte de héros " cosmogonique ", acteur d'une refondation du territoire.

Sans que l'on puisse vraisemblablement leur assigner une quelconque origine littéraire, ces versions expliquent la disparition finale de Cola par l'ordre royal qui lui est intimé d'aller examiner les fondations sous-marines de la Sicile. Ayant constaté que l'île repose sur trois piliers dont l'un est endommagé et un autre complètement détruit, le plongeur substitue son propre corps à ce dernier et se transforme en colonne humaine de manière à

livre des dieux. Celtes et Grecs II, Paris, Payot, $2^{\mathrm{e}}$ partie, chap. 5, "Les Fomoires et les Telkhines ", p. 541-574. Sur les entités marines de la mythologie grecque plus ou moins zoomorphes et monstrueuses, cf. Carla Costa, "La stirpe di Pontos ", Studi e Materiali di Atoria delle Religioni, 39, 1968, p. 61-100.

27. J'ai esquissé ailleurs une comparaison entre la légende de Cola Pesce et les mythes relatifs aux Telkhines : F. Delpech, "Le plongeon des origines : variations méditerranéennes ", Revue de l'Histoire des Religions, 217 (2), 2000, p. 203-255 (p. 233-234).

28. F. Delpech, "De saint Nicolas à Cola Pesce... " (cit. supra n. 5). B. Croce jugeait la comparaison «cervellotica »; G. Pitrè, op. cit., en faisait plus de cas. 
retarder l'inévitable engloutissement ${ }^{29}$. Ces versions où l'on voit se remobiliser d'antiques mythèmes méditerranéens - l'île sur pilotis, la légende rhodienne des Telkhines, la métamorphose d'Atlas en pilier montagneux supportant le ciel $^{30}$ - ne reproduisent cependant aucun texte classique. Elles semblent par contre réactiver, en lui conférant une dimension auto-sacrificielle, le vénérable mythe sud-est européen et eurasiatique du "plongeon cosmogonique " (réalisé par un démiurge hybride ou zoomorphe participant, en tant que subordonné du dieu créateur, à l'instauration et à la consolidation du territoire émergé ${ }^{31}$.

Cette devotio catapontique s'accompagne, dans plusieurs versions, de la découverte par le plongeur de l'existence d'un feu sous-marin, parfois mis en rapport avec l'activité cachée de l'Etna, et elle aboutit dans certains cas à sa mort par combustion. Ce réseau légendaire semble reconduire à une variante locale, attestée par ailleurs en Sicile, du cycle mythique indo-européen dit $\mathrm{du}$ "feu dans l'eau » comme j'ai essayé de le montrer dans un travail dont la présente étude est l'un des compléments ${ }^{32}$.

Les remarques qui précèdent permettent peut-être de rendre compte, autrement que par la supposition d'une fantaisie d'auteurs, de deux versions particulièrement atypiques de notre cycle qui, quoique relevant de son corpus écrit, reconduisent à une vision toute mythique de son protagoniste principal, par ailleurs absente des autres versions littéraires.

1/ - La première figure dans un texte grec du XVe ou XVI ${ }^{\mathrm{e}}$ siècle, dû à un anonyme compilateur byzantin peut-être originaire de Dalmatie ou d'Italie du Sud. "Nicolaos Poisson l'immortel ", " seigneur de toute la mer ", y est décrit comme une sorte de divinité marine capable de parcourir en une nuit tous les océans de la planète. Sa résidence permanente se trouve dans l'île fabuleuse de Gabalarada (dont l'emplacement n'est pas précisé) qu'il habite en compagnie de ses filles, lesquelles sont présentées comme des sirènes anthropomorphes blondes, lumineuses, parleuses, mais amphibies, ichthyophages et recouvertes d'une peau encore plus brillante que celle des poissons : ces créatures solaires (quoique nées en mer) font à l'occasion le bonheur des navigateurs qui parviennent à les capturer mais elles ne peuvent subsister hors de l'eau plus de 40 jours $^{33}$. Ce récit fait curieusement écho à la tradition arabe, développée dans les 1001 Nuits et plusieurs autres textes ${ }^{34}$, selon laquelle le conquérant arabe

29. Cf. G. Cavarra, op. cit., p. 120-123, 132-133, 138-141.

30. Sur les îles supportées par des piliers cf. B. Sergent, «Une Délos celtique ", Ollodagos, 19, 2005, p. 117-138. Pour la version ovidienne du mythe d'Atlas cf. Mét. IV, 657-662.

31. Cf. Mircea Eliade, De Zalmoxis à Gengis-Khan. Études comparatives sur les religions et le folklore de la Dacie et de l'Europe Orientale, Paris, Payot, 1970, chap. 3, p. 81-130, et F. Delpech, "Le plongeon des origines..." " cit. supra n. 27.

32. F. Delpech, « De saint Nicolas à Cola Pesce.. ", cit. supra n. 5.

33. Sur ce texte byzantin cf. Francesco Sbordone, Scritti di varia filologia, Naples, Giannini, 1971, chap. 7, p. 225-233, «Un’ eco bizantina della leggenda di Nicola Pesce », et G. B. Bronzini, op. cit., p. 364-366.

34. Sur cette légende arabe cf. Les Mille et une Nuits, trad. J. E. Bencheikh et A. Miquel, Paris, Gallimard (Pléiade), t. 2, p. 580 sq. (577 nuit) et Julia Hernández Juberías, La Península Imaginaria. Mitos y leyendas sobre Al-Andalus, Madrid, CSIC, 1996, p. 34, 40-42, 59. 
de l'Espagne, Mûsà b. Nusayr, au cours d'imaginaires pérégrinations à travers un mythique Occident (pén)insulaire en quête des vases de Salomon, aurait découvert, outre la fameuse Ville d'Airain, une peuplade primitive d'indigènes ichthyophages et troglodytes vivant au bord d'une sorte de lac ou de mer intérieure, tribu jadis convertie à l'Islam (et régulièrement visitée) par l'immortel prophète aquatique et verdoyant $\mathrm{Al}$-Khadir, qui lui aussi parcourt sans cesse les espaces marins ${ }^{35}$. Ce dernier se manifeste par des épiphanies maritimes, notamment liées à cette mer - où ont été immergés les vases salomoniens -, laquelle est précisément et également habitée par des femmes-poissons, que Mûsà, après leur capture, emmène avec lui à Damas pour les présenter au calife, sans pouvoir empêcher toutefois qu'arrachées à leur élément elles ne meurent bientôt, quoique l'on ait pris la précaution de les loger, comme Cola Pesce dans son tonneau, dans des cuves remplies d'eau...

2/ - L'autre texte qu'il y a lieu de prendre en compte est le romance vulgar espagnol de 1608, Relación de como el pece Nicolao se ha aparecido de nuevo en el mar ${ }^{36}$. Dans cette version hispanisée de la légende sicilienne, "Pece Nicolao" est dit natif de Rota, près de Cadix ${ }^{37}$. Dès l'âge de dix ans, sa passion pour la mer est telle que ses parents l'enferment pour la contrecarrer. En vain : «soltóse, y al mar acude / que no ay de la mar quitarle ". Alors qu'il s'apprête à se jeter dans les flots en s'exclamant "; $O$, quién fuera Pece!", son père le prend au mot et lance la fatale malédiction : "Pece te vuelvas [...] fuera de la mar no pares. I Mueras en saliendo d'ella». Métamorphose immédiate de l'enfant (« ...el medio cuerpo / vio de pescado espantable ") et disparition dans les flots, d'où on ne le voit ressurgir, plusieurs mois après, que pour de fugitives apparitions, au cours desquelles il vient près du rivage saluer les siens ou renseigner les gens de mer (qui en tirent "la carta del marear") sur les fonds et les passages périlleux. Suivent l'épisode des noces de la sœur, à laquelle Nicolao assiste dans son tonneau, puis une immersion beaucoup plus longue (de 100 années!), pendant laquelle Nicolao visite l'effroyable et tonitruante "cueva de Rota", gouffre sous-marin obscur, producteur d'un tourbillon dangereux pour les navires, d'où il n'est ressorti que l'hiver dernier pour rendre compte aux navigateurs de son expérience, qui fait l'objet d'un second romance. Dans ce rapport notre héros se dit homme et chrétien, se nomme ("El Pece Nicolao soy») et raconte

35. Cf. Françoise Aubaille-Sallenave, "Al Khidr, "L'homme au manteau vert" en pays musulmans : ses fonctions, ses caractères, sa diffusion ", in Res Orientales, vol. 14 (Charmes et sortilèges. Magie et magiciens), 2002, p. 11-35 (p. 19-22 pour ses affinités aquatiques). Ce personnage mythique apparaît dans les récits arabes médiévaux sur la préhistoire de l'Espagne et, à plusieurs reprises, dans la littérature aljamiada des Morisques.

36. Cf. l'édition de ce texte in M. D'Agostino, op. cit., p. 78-105 et l'analyse qu'en donne le même auteur (ibid., p. 56-75) qui établit un lien (discutable mais suggestif) entre cette tradition, la légende du messie ichthyomorphe des Marranes et la première continuation du Lazarillo. Les deux éditions originales de cette Relación (Salamanque et Barcelone) sont de 1608.

37. Plusieurs textes antiques font allusion à la présence d'" hommes marins " dans la baie de Cadix, non loin de laquelle se produisent les ichthyomorphoses du Messie marrane et de Lazarillo et l'apparition méridionale de l'" hombre pez de Liérganes" auquel croyait B. Feyjóo : cf. F. Delpech, «Lazare, l'eau, le vin et les thons... », cit. supra n. 9. 
son aventure : une fois entré dans la cueva il a parcouru pendant 40 jours un obscur tunnel sous-marin pour parvenir finalement à une sorte de lumineux et tranquille paradis maritime, dont on ne sait s'il est situé dans les profondeurs ou au-delà des frontières du monde connu. Cette mer intérieure à la mer et inaccessible aux hommes est "diáfana y clara / como el christalino espejo ", et ses rives rejoignent les eaux du Jourdain, lesquelles confèrent aux poissons jeunesse et vie éternelles ${ }^{38}$, ce qui leur évite notamment d'avoir à se reproduire et de se faire la guerre. Ils passent donc leur vie à exalter le créateur par le muet langage de leurs danses "en concertados rodeos ». De cette Mer Promise tranquille et heureuse, où Nicolao, garde " a pesar del gusto », quelque nostalgie du monde humain, notre "medio pescado ", doué de "razón y entendimiento ", est le seul seigneur ("todos los peces del mar / quiere Dios me estén sujetos"). Il pourrait même revendiquer un statut divin ("Soy el Neptuno del mar") si ne le retenait un reste prudent d'orthodoxie religieuse (".. pero yo nunca pretendo / ser como dios adorado / que a un Dios estoy sujeto "). Revenu donc plus près des hommes, il entreprend de faire part du savoir maritime qu'il a acquis à des navigateurs en difficulté en leur recommandant bien de coucher par écrit le "notable regimiento para navegar seguro " qu'il leur dicte. Les ayant remis sur leur droit chemin en les accompagnant " el medio cuerpo de fuera / y el otro medio en el agua ", il reprend ensuite ses pérégrinations solitaires. On l'aperçoit çà et là, notamment aux Bermudes ; mais, quoiqu'il s'adresse de loin en espagnol aux équipages des navires qu'il rencontre, beaucoup le redoutent "por no saber qué fuesse ". Son indécidable statut, son insaisissable nature continuent à alimenter la rumeur : "Unos dizen es Serena [...]. Otros dizen es demonio [...], otros que es hombre marino / otros dizen ser fantasma ". Sur les plages de Rota, où les descendants de son lignage sont encore installés, on attend toujours le retour de cette "octava maravilla »...

Dans cette fiction relevant de la paralittérature des pliegos de cordel nombre de détails reconduisent à la culture de l'écrit : Nicolao y est implicitement présenté comme immortel, à l'instar du Nicolaos du texte byzantin, et on peut même subodorer une (sans doute très indirecte) réminiscence ovidienne dans l'évocation du pieux ballet nautique des poissons ("Hablan con solo moverse / en concertados rodeos, / todo es dançar y dar gracias / al hazedor de los Cielos "), qui ne manque pas de rappeler la danse dionysiaque des dauphins, qui conclut le récit de la métamorphose des pirates tyrrhéniens, dont les évolutions chorales donnent corps à une version marine du dithyrambe («[...] inque chori ludunt

38. "No embejecen los pescados / ninguno muere de viejo / porque en el Jordán renacen [...]" (vv. 169-171, éd. M. D’Agostino, op. cit., p. 90-92). Cette notation fait partie du second romance de la Relación, qui contient un récit fait par Nicolao à des pêcheurs "en el día de la Circuncisión " : cette harangue porte-t-elle la trace d'une tradition rabbinique ? Cf. F. Delpech, « Du folklore au discours prophétique : le cas du Messie ichthyomorphe des Marranes ", in A. Redondo (éd.), La prophétie comme arme de guerre des pouvoirs (XVe-XVII siècle), Paris, Presses de la Sorbonne Nouvelle, 2000, p. 379-403, et M. D’Agostino, " ¿ Una fuente folclórica napolitana para la primera continuación del Lazarillo de Tormes ? ", in P. Civil et alii (éds.), Fra Italia e Spagna. Napoli crocevia di culture durante il Vicereame, Naples, Liguori, 2011, p. 225-236. 
speciem lascivaque iactant / corpora [...] $»)^{39}$. Mais, parallèlement, cette forme de la légende ne ressemble pas aux autres versions littéraires, latines et romanes, qui se concentrent toutes sur l'épisode, ici absent, de la coupe jetée à la mer par le roi et de la mort subséquente du plongeur, et elle s'apparente par contre aux versions d'origine orale par son insistance spécifique sur la malédiction parentale et sa mise en scène de l'épisode comique du tonneau ${ }^{40}$.

\section{De Glaucus à Cola Pesce}

Les deux versions qu'on vient d'examiner se distinguent donc des autres, tant littéraires qu'orales, en ce qu'elles font de Cola Pesce, explicitement ou non, un être immortel et récurrent, alors que partout ailleurs on assiste à sa mort ou à sa disparition définitive (voire à sa transformation en pilier sous-marin). Or cette caractéristique, jointe entre autres à l'unicité et à la semi-divinisation du personnage, recommande une comparaison avec le mythe de Glaucus, qui fait précisément l'objet d'un assez long développement dans le poème ovidien ${ }^{41}$.

On sait que ce dieu marin avait d'abord été lui aussi un être humain - un pêcheur béotien d'Anthédon - qui, suite à une métamorphose physique et mentale surnaturelle (par absorption du suc d'une herbe inconnue, dont il avait constaté qu'elle avait provoqué la résurrection des poissons qu'il avait déposés dessus), était devenu un être hybride, doté d'une abondante pilosité verdâtre, de larges épaules et de bras azuréens, de jambes tordues se terminant en nageoires ${ }^{42}$. Comme dans la légende sicilienne, la transformation du garçon est consécutive à son immersion dans les profondeurs de la mer, et elle résulte d'une impulsion soudaine et irrésistible. Celle-ci étant en l'occurrence immédiatement déclenchée par l'absorption du suc merveilleux. Cette dernière provoque en lui un impérieux désir de changer de nature (" [...] subito trepidare intus praecordia sensi / alteriusque rapi naturae pectus amore " ${ }^{43}$ ) qui l'amène à plonger aussitôt dans le golfe en saluant la terre d'un adieu définitif. La mystérieuse prairie ripuaire où cette foudroyante conversion a lieu est présentée par Ovide comme

39. Ovide, Met., III, 685 sq. Cf. les commentaires concernant ces vers dans l'appareil critique de l'édition A. Barchieri (Ed. Mondadori, Fondation L. Valla, tome 2, 2007, p. 233) ainsi que Gérard Capdeville, «Virgile, le labyrinthe et les dauphins », in Mélanges H. Le Bonniec, Bruxelles, Coll. Latomus n ${ }^{\circ} 201,1988$, p. 65-82. L'image d'un ballet sous-marin est évoquée dans la byline russe de Sadko, où l'on voit pour la première fois saint Nicolas se manifester au fond de la mer...

40. Cf. supra nn. 15 et 34. Sur la malédiction parentale cf. infra.

41. Ovide, Mét., XIII, 898-968, XIV, 1-74. Sur le(s) mythe(s) de Glaucus - Glaukos, cf. Marinella Corsano, Glaukos. Miti greci di personaggi omonimi, Rome, Ateneo, 1992, Bernard Deforge, "Le destin de Glaucos ou l'immortalité par les plantes ", in Visages du Destin dans les mythologies (Mélanges J. Duchemin), Paris, 1983, p. 21-39, et Ida Paladino, "Glaukos, o l'ineluttabilità della morte ", Studi Storico-Religiosi, II (2), 1978, p. 289-303. A. Sepilli, op. cit., p. 298-300, évoque Glaucus à propos de Cola Pesce mais, sans ébaucher une étude comparative, ne retient que leur commun assujettissement à la fatalité et leurs liens à l'Italie.

42. Ovide, Mét., XIII, 913-915 et 960-963.

43. Ibid., XIII, 945-946. 
un espace paradisiaque intact et inviolé, comme un fragment inconnu de l'Âge d'Or miraculeusement préservé et coupé du reste du monde, auquel aucun animal, aucun être humain n'avait encore eu accès avant que Glaucus n'y fût, comme par inadvertance, divinement téléguidé. Espace utopique, préfigurant les paradis ultramarins fréquentés par le Nicolaos du texte byzantin et le Pece Nicolao du romance espagnol. Cette prairie où croît l'herbe d'immortalité apparaît comme le complément, la porte et l'hypostase terrestre du monde divin des profondeurs auquel accède Glaucus par son plongeon au fond du gouffre, où l'accueillent les dieux de la mer. Par l'opération d'Oceanus et de Téthys, dans un état de transe semi inconsciente, Glaucus est en effet dépouillé de toute composante mortelle, purifié au moyen d'un carmen neuf fois répété, et il subit sur ordre divin une sorte de baptême cosmique en se voyant obligé de se plonger dans cent eaux courantes et de recevoir sur sa tête la masse aquatique déversée sur lui par les fleuves descendus pour ce faire de tous les points de la planète $^{44}$. Ce n'est qu'après cette opération, dont le rajeunissement sans fin par les eaux du Jourdain, évoqué dans le romance espagnol, peut passer pour la version (judéo)christianisée, que Glaucus reprend conscience et constate la définitive métamorphose physique et mentale dont il vient d'être l'objet ${ }^{45}$.

Quant à son cadre géographique, ses origines et ses circonstances, la métamorphose de Glaucus est certes différente de celle que subit Cola Pesce, et l'immortalité divine à laquelle il accède n'a d'équivalent que dans les versions atypiques de la légende sicilienne résumées plus haut. Cette dernière ne saurait donc être un simple démarquage du Glaucus de la mythographie.

Retenons cependant que les analogies entre ces deux traditions vont au-delà de celles que pourrait expliquer leur référence commune à la typologie-standard des récits de catapontisme ${ }^{46}$, ou que laisserait imaginer leur reconduction supposée à un fantomatique "inconscient collectif ", dont elles relèveraient chacune pour son propre compte ${ }^{47}$.

Il faut en effet remarquer que si la métamorphose marine de Glaucus a lieu en Béotie, c'est ensuite dans le détroit de Messine que se déroulent ses principales aventures, notamment ses amours malheureuses avec Scylla, transformée, on le sait, en monstre par Circé, jalouse de la passion qu'elle inspire à Glaucus et furieuse de se voir repoussée par ce dernier ${ }^{48}$. La célébrité de cette fable

44. "[...] nec mora, diversis lapsi de partibus amnes / totaque vertuntur supra caput aequora nostrum» (Ibid., XIII, 954-955).

45. Sur Glaucus chez Ovide cf. Dimitrios Matzilas, Les divinités dans l'œuvre poétique d'Ovide, Thèse Paris IV, 2000, p. 176-177.

46. Cf. Clara Gallini, "Katapontismos ", Studi e Materiali di Storia delle Religioni, 34, 1963, p. 61-90, Gustave Glotz, L'ordalie dans la Grèce primitive. Étude de droit et de mythologie, Paris, 1904, et F. Delpech, "Le plongeon des origines... ", cit. supra n. 27.

47. Ce postulat pseudopsychanalytique affaiblit la comparaison par ailleurs pertinente proposée par Marylène Possamaï, « Monstres marins du Moyen Âge » (cit. supra n. 18), p. 389-404.

48. Ovide, Mét., XIV, 1-74. Cf., sur l'italianisation du Glaukos crétois, M. Corsano, op. cit., chap. 4, p. 135-177, «Un cretese en Italia ». 
et son implantation sicilienne ont pu faciliter la surimpression et la mutuelle interpénétration du mythe grec de Glaucus et d'une légende locale.

Il est par exemple curieux de constater que la même allusion à une végétation sous-marine absolument identique à la végétation terrestre apparaît dans les deux traditions (sous forme d'adynaton paradoxal dans la version ovidienne du mythe de Glaucus, et de description des fonds sous-marins réels dans le texte médiéval de Gervais de Tilbury sur le nageur sicilien) ${ }^{49}$; réminiscence littéraire de la part du compilateur érudit ou références communes à un même système de croyances implanté depuis très longtemps dans l'imaginaire local $^{50}$ ? La seconde hypothèse me semble conseillée par les études récentes sur l'iconographie vasculaire et funéraire de l'Italie du Sud et de la Sicile antiques, qui révèlent l'importance qu'y ont eue les thèmes de l'hybride marin et du "plongeon initiatique ", et leur probable lien avec une imagerie des entités psychopompes et de l'accès aquatique à l'au-delà ${ }^{51}$.

Autre analogie remarquable entre nos deux hybrides : leur aptitude prophétique. Don divinatoire que reçoit Glaucus (dont Virgile fait le père de la Sibylle de Cumes) en même temps que l'immortalité et le statut divin ${ }^{52}$; quant à l'aptitude de Cola Pesce à prévoir les tempêtes - dont il avertit les navigateurs - elle est plutôt liée à sa connaissance intime de l'univers marin. Il s'agit bien cependant, de part et d'autre, de deux variations sur les thèmes mythiques indo-européens du savoir surnaturel des "Vieux de la mer " et du principe immanent de Vérité détenu par les Eaux et leurs habitants, qui en fait l'élément et les acteurs privilégiés des ordalies juridiques et royales ${ }^{53}$.

Il y aurait sans doute lieu de s'interroger aussi sur la possible parenté entre le tonneau de Cola Pesce (appelé curieusement "Nicola Pipe » dans les textes de W. Map et G. de Tilbury) et la jarre pleine de miel où tombe et se noie (pour ressusciter ensuite) le Glaukos de la version crétoise du cycle de mythes grecs concernant des personnages portant ce même nom ${ }^{54}$. L'immersion dans un

49. Ovide, Met., XIV, 37-39 (à comparer avec l'évocation des forêts sous-marines dans les versions de G. de Tilbury et P. Bersuire : cf. supra n. 18).

50. Cf. J. Leclercq-Marx, op. cit.

51. Cf. Paloma Cabrera Bonet, "Del mar y sus criaturas. Seres híbridos marinos en la iconografía suritálica ", in I. Izquierdo et H. Le Meaux (éds.), Seres Hibridos. Apropiación de motivos miticos mediterráneos, Madrid, Casa de Velázquez, 2003, p. 111-139. Sur la « tombe du plongeur » de Paestum, cf., entre autres, Daisy Warland, «Tentative d'exégèse des fresques de la "tombe du Plongeur" " de Poseidonia ", Latomus, 57, 1998 (1), p. 261-291.

52. Cf. Henri Jeanmaire, Couroi et Courètes, Bibl. Univ. de Lille, 1939, p. $444-450$ ("Le conte de Glaucos »), M. Corsano, op. cit., chap. 1, p. 11-36 ("Glaukos dio marino. La diversa oracolarità di due divinità omonime : Glaukos di Antedone e Glaukos di Corinto »). Sur les aspects oraculaires des dieux marins protéens dans le monde indo-européen, cf. C. Sterckx, op. cit., passim, et D. Briquel, op. cit.

53. Cf. Marcel Détienne, Les Maîtres de Vérité dans la Grèce archaïque, Paris, Maspéro, 1967, D. Briquel, op. cit.

54. Sur ce Glaukos, fils de Minos, cf. M. Corsano, op. cit., chap. 3, p. 111-134 («Glaukos figlio di Minosse. L'iniziazione di un bimbo caduto nel miele ") : l'auteur discute la thèse de B. Deforge (op. cit.) selon laquelle les trois principaux « Glaukos » sur lesquels il existe des légendes (comportant des thèmes communs) seraient en fait un seul et même personnage (p. 188-190). 
récipient rituel - lui-même équivalent symbolique du gouffre ou "chaudron " marin $^{55}$ - actualise le passage provisoire par la mort, laquelle est finalement conjurée ou abolie par une renaissance et par l'accès à un nouveau statut. Elle trouve donc naturellement sa place dans le scénario initiatico-divinatoire dont nos deux héros semblent parallèlement tributaires, comme le sont par ailleurs les autres Glaukos du cycle hellénique relatif à ces homonymes ${ }^{56} \ldots$

Il est d'ailleurs significatif que le passage de l'obscurité à la lumière, qui est consubstantiel à ce cycle mythique et à l'étymologie même du nom commun que partagent ses héros ${ }^{57}$, soit aussi au centre des versions de la légende de Cola Pesce, dans lesquelles ce dernier gagne l'immortalité, notamment dans celle de la Relación espagnole, où il traverse pendant 40 jours un "callejón estrecho " totalement obscur avant de trouver sa mer intérieure « diáfana y clara / como el christalino espejo $"^{58}$.

On retiendra enfin que la métamorphose de Glaucus et celle du nageur sicilien partagent une caractéristique qui les distingue de la plupart des autres métamorphoses du corpus ovidien : elles sont parfaitement ambivalentes. Non seulement parce qu'il s'agit de métamorphoses seulement partielles qui créent des entités hybrides, voire amphibies, lesquelles restent humaines dans leur (quasi)animalité ou leur divinité supposée, ou parce que des facteurs naturels et surnaturels s'associent dans le processus de leurs transformations respectives. Aussi et surtout pour deux autres raisons :

1/ - leur destin, y compris lorsqu'ils atteignent l'immortalité, est à la fois heureux et malheureux. En devenant homme marin Cola assouvit enfin une passion profonde ; on a vu que, au moins dans deux versions, il accède même à un royaume paradisiaque dont il devient le maitre et quasiment le dieu. Cette promotion fusionnelle n'est cependant que la face positive d'un fatum subi qui l'exclut de la communauté humaine, dont il ressent et avoue périodiquement la nostalgie, et dans la plupart des versions il connaît une fin tragique, éventuellement auto-sacrificielle, lorsqu'il ne peut s'empêcher de céder au caprice royal ou de s'immerger et pétrifier à jamais pour retarder le désastre géologique qui menace inexorablement la Sicile. Le Glaucus d'Ovide n'est pas plus heureux, qui se plaint amèrement de ce que son statut divin ne suffit pas à lui concilier l'amour de Scylla, qu'il aura finalement la douleur de voir transformée en monstre. Dans certaines versions son inutile immortalité devient même malédiction, puisqu'il y est précisé qu'elle ne s'accompagne pas d'une perpétuelle jeunesse et le condamne donc à un vieillissement sans fin qui le rend de plus en plus décrépit et méconnaissable : dans ce cas, comme dans quelques autres variantes du cycle de Glaukos, le récit s'apparente indirectement aux mythes admonitoires proche-orientaux sur l'inéluctabilité de la mort ou

55. Cf. A. Seppilli, op. cit., p. 187-216.

56. Cf. H. Jeanmaire, op. cit., loc. cit., et M. Corsano, op. cit., p. 190 sq.

57. Ibid., p. 129-130 (à propos des sens de l'adjectif "glaukós»).

58. Éd. M. D’Agostino (in La leggenda di Cola Pesce, cit. supra n. 2), p. 90 ("La oscuridad me afligí / de aquel callejón estrecho, / vi su remate y llegué / a ver del Sol los reflexos »). Dans la version byzantine les filles de Nicolaos se caractérisent par leur brillante luminosité. 
sur les graves inconvénients qu'encourt l'homme à vouloir lui échapper, voire à y réussir ${ }^{59} \ldots$

2/ - Le second signe de l'ambivalence profonde qui affecte les métamorphoses marines de Glaucus et de Cola Pesce est le caractère équivoque, voire contradictoire, des évaluations morales et interprétations allégoriques qu'elles suscitent.

On sait que dans la tradition poétique médiévale Glaucus jouit généralement d'une appréciation favorable, liée au thème éminemment positif de sa déification : Dante (Paradis I, 67-72) fait de sa métamorphose une sorte de préfiguration ineffable de sa propre expérience mystique et de la transformation interne opérée par Dieu en l'homme ${ }^{60}$, et l'Ovide moralisé (XIII-XIV) réinterprète dans une perspective christologique la résurrection des poissons, la descente rédemptrice du pêcheur dans la mer de ce bas-monde et la dimension baptismale de sa purification sous-marine ${ }^{61}$.

C'est en revanche une image toute négative du même personnage qui transparaît dans les traités de mythographie et dans la tradition emblématique. Cette image tenace remonte (au moins) à Fulgence et c'est Boccace qui, dans ses Genealogiae deorum gentilium, s'est employé à la recycler et l'a transmise aux mythographes ultérieurs ${ }^{62}$. Glaucus, en tant qu'amoureux de Scylla, y est censé représenter la luxure, et l'étymologie de son nom (supposé évocateur d'un défaut de vision) est convoquée pour associer à une forme de cécité mentale la passion incontrôlée qui y conduit. Ce qui n'empêche pas l'auteur, se fondant alors sur une autre étymologie (qu'il attribue à Leonzio Pilato) selon laquelle le même nom évoquerait en fait la terreur, de proposer dans un autre chapitre une explication différente de la signification du mythe : les liens contradictoires de Glaucus à Circé et à Scylla seraient une extrapolation fabuleuse de la terreur inspirée par les hurlements fracassants des courants marins heurtant les écueils et s'engouffrant dans les cavernes subaquatiques qui abondent dans les lieux

\footnotetext{
59. Cf. I. Palladino, op. cit.

60. Cf. Robert Hollander, Allegory in Dante's Commedia, Princeton Univ. Pr., 1969, p. $217-$
} 232.

61. Ovide Moralisé (éd. C. de Boer), t. IV, p. 471-478. Cf. M. Possamaï, op. cit., et ead. (M. Possamaï-Pérez), L'Ovide Moralisé. Essai d'interprétation, Paris, Champion, 2006, p. 460461 et 527 sq.

62. Boccaccio, Genealogie deorum gentilium, V. Zaccaria (éd.), Milan, Mondadori, 1998 (= tomes 7-8 de l'éd. des CEuvres Complètes par V. Branca), vol. 1, p. $399-403$ (livre IV, chap. 14), et p. 983-989 (livre X, chap. 9). On retrouve les mêmes interprétations (qui procèdent de Fulgence) dans la Philosophia secreta de Juan Pérez de Moya (cf. livre II, chap. 13, " De los peligros del mar ») : j'utilise une (médiocre) édition moderne (Barcelone, Glosa, 1977, 2 vols., t. 1, p. 129 sq.). On peut suivre également la trace de Glaucus dans la littérature emblématique, qui, à la suite d'Alciat (Emblemata $\mathrm{n}^{\circ} 26$ ), en donne généralement la même interprétation négative, et dans la poésie bucolique (cf. la 7 églogue de La Bucólica del Tajo de Francisco de la Torre, p. 275-279, in idem, Poesía completa, M. L. Cerrón Puga, éd., Madrid, Cátedra, 1984, dont la tonalité est essentiellement lyrico-élégiaque, le dieu marin exprimant à la première personne la souffrance que lui cause son amour non partagé pour Scylla). 
hantés par ces deux femmes fatales ${ }^{63}$. Il est intéressant de constater que le même télescopage d'un paradigme optique et d'un code acoustique - d'ailleurs caractéristique du cycle mythique indo-européen du "feu dans l'eau " ${ }^{64}$ apparaît aussi dans le romance espagnol, où l'aventure sous-marine du "Pece Nicolao " commence dans l'obscurité de la " cueva de Rota ", célèbre pour le fracas tonitruant qu'y produit la mer ("Da bramidos el mar [...] que se oyen treinta leguas $")^{65}$.

La métamorphose de Cola Pesce n'a certes pas fait l'objet d'autant d'interprétations allégoriques que celle de Glaucus, mais on y remarque la même ambivalence.

Il apparaît souvent, lui aussi, comme un personnage positif: plusieurs auteurs notent les bienfaits qu'il prodigue aux navigateurs par ses enseignements et avertissements et certaines versions orales en font, on l'a vu, un héros sacrificiel qui sauve provisoirement la Sicile d'un engloutissement annoncé. Le plus ancien des textes qui le mentionnent, une strophe du troubadour Raimon Jordan, le présente comme un sage virtuel ("que si visques lonc temps savis hom fora ") ${ }^{66}$, l'identifie implicitement à saint Nicolas (il l'appelle " Nicola de Bar ») et l'institue comme une sorte d'allégorie courtoise de la fidélité amoureuse et du sort fatal consenti auquel elle voue le poète. On assiste même au XIVe siècle à une ébauche de glose spirituelle du personnage dans le Reductorium morale de Pierre Bersuire, qui fait écho à celle de Glaucus dans l'Ovide moralisé : ses investigations sous-marines sont comparées à celles des théologiens dans les "profunda maris scripturarum", où ils trouvent les "dulcia flumina scientiarum divinarum $"{ }^{67}$. L'imagerie médiévale associant le monde marin aux mystères de la religion, laquelle remonte au moins à Basile de Césarée et Ambroise de Milan et plonge ses racines à travers le judaïsme dans un très ancien symbolisme sémitique $^{68}$, suffit à expliquer ce tour inattendu pris par l'évaluation de notre nageur sicilien : c'est la référence commune à cet arrière-plan culturel et à son implantation privilégiée dans la région jadis phénicienne de Cadix qui justifie les comparaisons que l'on a pu faire entre cette légende et celle du Messie

63. Boccaccio, op. cit., p. 399 sqq. (livre IV, chap. 14), en particulier p. 403. Comparer avec le discours du prince Guillaume sur la transformation temporaire des chevaliers chrétiens en poissons par la magicienne païnne Armide dans la Jérusalem délivrée (X, 66-67) du Tasse.

64. Cf. F. Delpech, "Saint Nectan : hagiographie, folklore et mythologie comparée ", in G. Hily et alii (éds.), Deuogdonion (Mélanges C. Sterckx), Rennes, Tir (Publ. du CRBC Rennes 2, Univ. Europ. de Bretagne), 2010, p. 133-155. Je reviendrai ailleurs sur les liens des traditions sur saint Nectan (et saint Neot) avec la légende de saint Nicolas.

65. Éd. M. D’Agostino, La leggenda..., p. 86-87.

66. Ibid., p. 110-111 (et p. 23-24 pour le commentaire).

67. Ibid., p. 114-115 (p. 29-30 pour le commentaire).

68. Cf. F. Delpech, « Du folklore au discours prophétique... », cit. supra n. 38, et in F. Delpech (éd.), Limaginaire des Espaces Aquatiques en Espagne et au Portugal, Paris, Presses de la Sorbonne Nouvelle, 2009, l'article de Suzy Béramis ( Le voyage de Jonas, figure de l'expérience mystique chez saint Jean de la Croix ", p. 23-45) et l'introduction de F. Delpech ("Éléments pour une poétique ibérique des espaces aquatiques", p. 9-20, en particulier p. 15-16). 
ichthyomorphe attendu au XVI ${ }^{e}$ siècle par les Marranes andalous ${ }^{69}$, ou celle de l'une et de l'autre avec l'aventure sous-marine de Lázaro transformé en thon dans la continuation anversoise de $1555^{70}$, sans qu'il soit nécessaire de supposer une quelconque corrélation ou interdépendance conjoncturelle entre ces trois traditions ${ }^{71}$.

Cette image positive de Cola Pesce, que certains traits de sa légende rattachent à saint Nicolas, est cependant contrebalancée par l'interprétation funeste de sa métamorphose que laissent transparaître certaines versions. Cola lui-même, dont il est précisé parfois qu'en mer il ne montre que la partie supérieure, entièrement humaine, de son corps, laissant immergée la partie zoomorphe, semble conscient et comme honteux de ce versant négatif ${ }^{72}$. Plusieurs versions indiquent, plus ou moins clairement, que sa métamorphose est le fruit d'un châtiment. C'est sa désobéissance à ses parents, son excessive tendance contrenature à se séparer de la société des hommes pour rechercher la solitude du milieu marin, que sanctionne cette transformation, qui rend cet isolement quasi total et définitif. Ce trait est souligné dès la première version complète de la légende, celle de Salimbene de Adam, qui conclut son récit en indiquant que l'enfant " offendit graviter et exasperavit matrem " et cite à ce propos l'admonition de l'Ecclésiaste : "Quam male fame est qui relinquit patrem, et est maledictus a Deo qui exasperat matrem ${ }^{73}$.

Ce motif peccamineux est repris plus laïquement et élargi par Pontano qui, dès le premier chapitre de son De immanitate liber, prend «Cola Pisce» comme exemple de la déshumanisation de ceux qui s'étant écartés de la voie moyenne de la vertu et des " honesta officia " finissent par perdre la raison et leur nature humaine et "ad feras transeunt $»^{74}$. Sur ce point, tradition littéraire et corpus folklorique oral ont coïncidé puisque, selon un souvenir d'enfance cité par Pipino da Bologna dans son Chronicon, "Nicolaus Piscis " était mentionné par les mères pour terroriser leurs enfants vagissants et les rappeler à l'ordre ${ }^{75}$. Le même message est transcrit, avec plus d'onction, dans l'édition salmantine du romance de 1608, laquelle est accompagnée de deux canciones invitant expressément et respectivement les parents à veiller à ce que leur progéniture soit «bien acostumbrada » et les enfants à suivre "la bondad y el camino de la gloria ", c'est-à-dire avant tout à honorer leurs parents et à leur obéir ${ }^{76}$.

69. F. Delpech, «Du folklore au discours prophétique... », cit. supra n. 38.

70. Id., "Lazare, l'eau, le vin et les thons... ", cit. supra n. 9.

71. M. D’Agostino, "¿ Una fuente folclórica napolitana... ? ", cit. supra n. 38, qui insiste à juste titre sur la symbolique chrétienne qui traverse la Relación de 1608.

72. "El medio cuerpo de fuera / y el otro medio en el agua " (Relación..., éd. M. D’Agostino, La leggenda..., p. 100). Cf. l'article de Claudie Balavoine in L'imaginaire des Espaces Aquatiques..., op. cit. supra n. 68, ainsi que M. Possamaï-Pérez, L'Ovide Moralisé, op. cit. supra n. 61, p. 527-528.

73. M. D’Agostino, La leggenda..., p. 110.

74. I. I. Pontani, De immanitate liber, op. cit. supra n. 10, p. 5-6.

75. M. D’Agostino, La leggenda..., p. 112.

76. Ibid., p. 138-139. 
La négativisation de notre personnage atteint enfin son terme ultime dans les versions bretonnes où « le poisson Nicole " n'a plus rien d'humain et passe son temps à jouer de mauvais tours aux pêcheurs, au point qu'il faut parfois le faire exorciser par un prêtre 7 .

On voit donc que Glaucus et Cola Pesce, au-delà de leurs analogies intrinsèques, se rejoignent dans l'équivocité des interprétations qui ont pu être proposées quant à leurs métamorphoses. Le reflet le plus marquant de cette ambivalence partagée est l'incertitude relative à leurs statuts qu'ont parfois affichée les auteurs qui se sont intéressés à leurs légendes respectives : le doute auquel fait allusion la Relación espagnole quand elle évoque les multiples rumeurs circulant sur "El Pece Nicolao " (est-il une sirène, un démon, un homme marin, un fantôme ?) semble faire écho à l'inquiétude qu'Ovide attribue à Scylla lorsqu' elle voit paraître devant elle cet être indéfinissable qu'est Glaucus " monstrumque deusne / ille sit ignorans» (Met., XIII, 912-913).

\section{La mère coupable et la Déesse Syrienne}

Au terme de cette ébauche de comparaison entre le dieu marin évoqué par Ovide (entre autres) et le nageur prodigieux de Messine, on peut se demander, compte tenu de leurs nombreuses analogies, si le second n'est pas, dans une certaine mesure, le produit d'une "survivance " du premier, dont le souvenir se serait maintenu et transmis dans la tradition orale, le mythe méditerranéen devenant, moyennant quelques déménagements et adaptations à de nouveaux contextes, la légende locale imparfaitement christianisée que nous voyons surgir dans la littérature écrite vers la fin du XII siècle. Relation généalogique donc, ou purement typologique ? À moins que leurs analogies ne s'expliquent par un héritage commun dont l'un et l'autre seraient séparément tributaires ? Ces diverses hypothèses ne s'excluent pas nécessairement, sauf à les envisager dans une perspective excessivement totalitaire.

Les différences entre les deux personnages sont en effet aussi instructives que leurs analogies. Contrairement à Glaucus, Cola n'est pas un dieu et il n'est dit immortel (ou capable de se rajeunir à volonté) que dans deux versions paralittéraires, bien distinctes du reste du corpus. Ce n'est pas en tant que pêcheur (ou fils de pêcheur), mais en tant que nageur et plongeur exceptionnel, qu'il est amené à fréquenter l'élément marin pour finalement s'y fondre et y changer d'aspect. Entre le mythe et la légende il y a donc divergence de plan.

L'hypothèse d'une transition possible de l'un à l'autre est cependant validée par le fait que, bien avant Ovide, on voit apparaître - dans les Histoires incroyables de Palaephatos - une version grecque de l'histoire de Glaukos où ce dernier n'a rien d'un dieu et doit précisément sa réputation légendaire à ses dons de nageur et de plongeur, lesquels lui permettent de disparaître longtemps

77. P. Sébillot, op. cit., loc. cit., et P. J. Hélias, op. cit., loc. cit. Cf. aussi A. Seppilli, op. cit., p. 319-320. 
et de faire croire à ses concitoyens qu'il dispose d'une résidence sous-marine, fable ensuite accréditée définitivement lorsqu’il disparaît pour de bon, victime de quelque monstre marin ${ }^{78}$. L'idée d'une imposture reste implicite dans le texte de Palaephatos, qui fait, ici comme ailleurs, œuvre de démystificateur rationaliste : n'est-elle pas cependant subliminalement latente dans les versions du mythe qui indiquent que Glaukos aurait choisi de s'immerger définitivement par désespoir de n'être pas parvenu à convaincre ses contemporains qu'il était effectivement devenu immortel ${ }^{79}$ ? Le catapontisme délibéré de Cola Pesce ne résulte certes pas d'un projet théologique aussi tordu, mais on voit comment l'anecdote pseudo-historique peut se dégager du mythe sous-jacent, l'apothéose marine surnaturelle du héros métamorphosé se fondant subrepticement dans le répertoire sportif d'une nomenclature de records nautiques.

Deux différences majeures séparent néanmoins la légende de Cola Pesce du mythe de Glaucus et confèrent à la première son irréductible originalité. La tradition sicilienne est d'une part obscurément traversée par le cycle hagiographique de saint Nicolas, dont elle transpose certaines composantes. Ayant abordé ce dossier ailleurs je n'y reviendrai pas ici. D'autre part la métamorphose du héros y est déterminée par une cause spécifique, la malédiction parentale (le plus souvent maternelle) consécutive à l'exaspération face à son hydrophilie obsessionnelle. Mentionné pour la première fois dans la chronique de Salimbene de Adam (XIII ${ }^{\mathrm{e}}$ siècle), le trait réapparaît dans quelques textes littéraires ultérieurs et dans la plupart des versions orales. Cette malédiction voue parfois expressément le garçon à ne plus pouvoir vivre hors de l'eau, voire à devenir (au moins partiellement) poisson.

Il est certes évident que l'effet des imprudentes exclamations de la mère irritée outrepasse largement ses intentions : une fois proférée dans la fureur, la parole malédictive se trouve dotée d'une efficacité magique imprévue, imparable et irréversible. Son effet est, en négatif, équivalent à celui du carmen solennel par lequel les dieux marins confèrent à Glaucus en le purifiant de son humanité sa nouvelle nature de divinité hybride.

Cette notion d'une puissance magique de la parole imprécatoire, manifeste héritage païen, est restée assez vivante au Moyen Âge pour alimenter tout un cycle de récits exemplaires, à la fois admonitoires et édifiants, mettant notamment en garde les parents contre les execrationes et autres jurons blasphématoires qu'ils

78. Palefato, Storie incredibili, A. Santoni (éd.), Pise, E.T.S., 2000, p. 83-85 (n 27, "Glauco del mare "). Sur Palaephatos cf. Paul Veyne, Les Grecs ont-ils cru à leurs mythes ? Paris, Seuil, 1983, p. 77 sqq. Remarquer que, dans le chapitre consacré à Glaucus par Baltasar de Vitoria (Primera parte del Teatro de los dioses de la gentilidad, Madrid, Imprenta Real, 1676, Livre III, chap. 14, p. 298-302), on assiste à une sorte de tentative de conciliation entre la version ovidienne, largement citée, et un point de vue démystificateur analogue à celui de Palaephatos : le pêcheur y est aussi un nageur prodigieux, dont les immersions prolongées auraient accrédité la fable de sa métamorphose divine. On notera surtout que l'auteur, pour justifier la crédibilité des exploits nautiques qu'il attribue à Glaucus, fait longuement référence, à titre comparatif, à l'histoire de Cola Pesce, "al qual algunos llaman Pez Colaz, y otros el Pece Nicolao ", qu'il connaît manifestement par plusieurs sources littéraires (Alessandri, Mexía, Textor, Pontano, etc.).

79. D’après une scholie à Platon, République : cf. B. Deforge, op. cit., p. 26. 
ont trop tendance à déverser sur leurs enfants désobéissants, et recommandant à ces derniers, particulièrement exposés au démon, d'éviter de susciter la colère de leurs géniteurs, que Satan est tout disposé à prendre au mot. Nombre d'histoires d'enfants inopinément "voués au diable » par l'incontinence verbale de leurs parents exaspérés se soldent ainsi par une effective, contraignante et immédiate devotio démoniaque, rendue possible par la profération de mots interdits qui automatiquement enchaînent ${ }^{80}$. L'enfant maudit par l'un ou l'autre de ses parents tombe immédiatement sous la coupe du démon qui en prend possession et il ne peut généralement être rédimé que par l'intervention de la Vierge.

On connaît les racines hagiographiques de ce genre de récit $^{81}$, lequel apparaît comme une version christianisée du cycle de contes folkloriques et de légendes initiatiques où divers scénarios sont mis en œuvre pour rendre compte du fait qu'à partir d'un certain âge les garçons doivent être enlevés à leurs parents consentants et conduits en forêt pour y subir des rites de passage sous la direction d'officiants spécialisés ${ }^{82}$. Lorsque ce système rituel archaïque a été contesté, pour être finalement aboli et remplacé par d'autres procédures d'intégration sociale et d'accès au statut d'adulte, ces récits vénérables mais désormais obsolètes ont dû être refondus afin d'apparaître encore intelligibles et moins scandaleux : s'est imposée alors l'idée plus acceptable d'une faute parentale préalable comme cause déterminante plus ou moins directe de la violence imposée à l'enfant, dont l'indiscipline occasionnelle ou récurrente fera figure de cause circonstancielle. Les prescriptions de l'Église concernant le bannissement des jurons, malédictions et autres formules incantatoires, réputées magiques et païennes mais toujours obscurément considérées comme efficaces, donc dangereuses, ont alors pu donner corps et intelligibilité au cycle d'exempla admonitoires dont manifestement relève l'histoire de la malédiction maternelle contraignante dont est victime Cola Pesce ${ }^{83}$. Ainsi était-il possible, dans un cadre mental d'inspiration chrétienne, de rendre compte de ce phénomène fulgurant et singulier de l'immédiate immersion, couplée à une métamorphose de l'enfant, que le mythe de Glaucus expliquait par une mystérieuse urgence de changer de nature, due à l'absorption d'une substance magique et par l'intervention cérémonielle des dieux de la mer.

80. Cf. Frédéric C. Tubach, Index Exemplorum. A Handbook of Medieval Religious Tales, Helsinki, F.F.C. 204, 1969, n 1440 ("Daughter cursed »). Cf. dans la version de Fazio degli Uberti l'avertissement aux parents qui maudissent leurs enfants (M. D'Agostino, La leggenda..., p. 112).

81. Cf. Paul Meyer, "L'enfant voué au diable ", Romania, 23, 1904, p. 163-178, et Guy de Tervarent et Baudouin Gaiffier, "Le diable voleur d'enfants ", in Mélanges Antoni Rubió i Lluch, Barcelone, 1936, t. 2, p. 33-58.

82. Cf. Vladimir J. Propp, Les racines historiques du conte merveilleux, trad. fr., Paris, Gallimard, 1983, p. 103-111.

83. Cf. la relación étudiée par Augustin Redondo in «Le diable et le monde diabolique dans les relaciones de sucesos (Espagne, $1^{\text {re }}$ moitié du XVII siècle) ", in J. P. Duviols et A. Molinié-Bertrand (éds.), Enfers et damnations dans le monde hispanique et hispano-américain, Paris, PUF, 1996, p. 131-158. 
En deçà du type de récit médiéval édifiant, normativement balisé et codifié, auquel reconduit formellement l'épisode en question, il est cependant loisible d'entrevoir en filigrane l'affleurement d'un autre modèle imaginaire et narratif qui par percolation en infléchit le cours et le sens. Le récit du catapontisme soudain et de l'hybridité consécutive de Cola, qui ne mobilise en rien le merveilleux chrétien, recèle trop de bizarreries et de résonances païennes pour faire sans plus figure d'exemplum admonitoire. L'insistance sur le rôle négatif ( ?) et déterminant de la mère sur le destin du héros - lequel est aussi l'accomplissement d'une vocation personnelle profonde et apparemment innée - est même en contradiction avec un thème récurrent du cycle chrétien de l'enfant voué au Diable suite à sa malédiction par la mère, selon lequel la possession est réversible et finalement annulée car seul le père peut juridiquement livrer son enfant à un autre tuteur ${ }^{84}$.

Par contre notre légende sicilienne est conforme, sur ce point, à toute une série de traditions orales (notamment ibériques) relatives à l'origine de telle ou telle sirène : ces créatures marines y sont généralement présentées comme des filles qui, d'abord "terriennes ", ont été un jour maudites par leurs mères, pour avoir commis telle ou telle faute, ou manifesté une excessive hydrophilie, et sont consécutivement devenues femmes-poissons ${ }^{85}$. Ces transformationos sont sans retour ni rédemption : à la différence des récits " exemplaires " mentionnés plus haut qui en ont fourni le cadre narratif, ces légendes qui ne font intervenir aucun démon ne visent plus tant à mettre en garde contre les dangers des incantations païennes qu’à souligner la force du lien matrilinéaire, manifestée par la puissance magique, seule efficace, de la parole maternelle et par l'influence décisive de la mère sur la nature et le destin de sa progéniture ${ }^{86}$.

De la mère à la mer, l'immersion apparaît donc dans ces traditions comme un approfondissement, par irrépressible retour aux eaux amniotiques, de la dépendance utérine de l'enfant. Ce dernier n'a plus dès lors qu'à s'enfermer plus ou moins définitivement dans le foetal paradis sous (ou ultra)-marin que s'approprie le Nicolao-Nicolaos de la Relación espagnole et de la version byzantine, ou à fatalement périr au fond du gouffre sur ordre de la substitutive figure paternelle que représente le roi abusif (dont plusieurs versions orales précisent, sans l'expliquer, qu'il souhaite la mort du jeune nageur $)^{87}$.

84. P. Meyer, op. cit., p. 166.

85. Cf. J. Caro Baroja, Algunos mitos españoles, op. cit. supra n. 17, loc. cit., et l'introduction de J. M. Pedrosa à El libro de las Sirenas, J. M. Pedrosa (éd.), Almería, Ayunt. de Roquetas de Mar, 2002, p. 37-42, "La maldición de las Sirenas (entre Melusina y el Pez Nicolás) ».

86. Cf. F. Delpech, "La légende de Dona Marinha : mythologie et généalogie », Cuadernos de Estudios Gallegos, LV (n 121), 2008, p. 407-426.

87. G. Cavarra, op. cit., p. 115-116 : le roi promet sa fille et son royaume à Cola Pesce s'il réussit pour la troisième fois à rapporter la coupe jetée à la mer ; le plongeur y parvient mais le roi le fait assassiner... Même scénario dans la version suivante (p. 116-118), mais Cola, dont le corps a été jeté dans le détroit, semble survivre (ou ressusciter ?) et il est toujours là, à surveiller la "ciumara di focu " qui menace les fondements sous-marins de la Sicile, où il est nourri par les sirènes dont les chants le maintiennent dans un état de perpétuelle jeunesse : ces versions folkloriques montrent que les fictions atypiques du texte byzantin et de la Relación espagnole ne 
La légende de Cola Pesce recoupe donc sur ce point, en lui offrant un contrepoint masculin, une notable partie du corpus traditionnel sur les sirènes, comme l'atteste notamment la symétrie entre la Relación de 1608 et un autre romance de cordel, intitulé "El fenómeno pez-mujer o la maldición de una madre", où sont contées les aventures d'une fille de Gerona qui, maudite par sa mère suite à une calomnie concernant ses mœurs, disparaît dans la mer, d'où elle ne réapparaîtra que sous la forme d'un monstre marin ${ }^{88}$.

Nous avons donc affaire, au-delà de toute influence ou récupération ecclésiastique et des schémas édifiants de la littérature des exempla, à un type de récit spécifiquement lié au monde marin et à ses habitants supposés. L'association de l'immersion et de l'ichthyomorphose à une transgression préalable concernant le rapport du sujet concerné avec ses ascendants, notamment sa mère, n'est pas une invention de clerc : elle se trouve déjà dans les mythes antiques de catapontisme, où, comme l'a souligné $\mathrm{C}$. Gallini, ce phénomène procède souvent d'un accès de folie déterminé par une crise familiale, elle-même fondée sur une faute (ou une série de fautes) - généralement de caractère sexuel commise par, ou contre, la mère (ou moins fréquemment un autre parent), folie qui se traduit par une pulsion suicidaire du contrevenant qui l'amène irrésistiblement à se précipiter dans la mer ou dans un fleuve. Toute une série de mythes et de légendes helléniques associent cette immersion spontanée, qui peut être à la fois symptôme maniaque et rituel thérapeutique ou salvateur, soit à l'intervention agressive d'une entité maternelle, laquelle peut se voir ellemême atteinte par cette folie consécutive à une transgression, soit à une faute (curiosité sacrilège, inceste, matricide) commise à l'encontre de cette entité.

On trouvera donc alternativement des histoires d'enfants jetés à l'eau par leur mère - laquelle peut s'y jeter avec eux -, comme il arrive dans les mythes d'Eumolpe ou d'Ino Leucothea ${ }^{89}$, de fils précipités dans la mer, ou se suicidant par noyade, après avoir causé un tort à leur génitrice ${ }^{90}$, ou encore de femmes (ou déesses) se précipitant avec leur progéniture dans un cours d'eau pour échapper à un viol ${ }^{91}$.

Le cas d'Ino, qui devenue folle, se jette à la mer avec son fils Mélicerte, retient ici l'attention, non seulement parce qu'il a été développé par Ovide ${ }^{92}$, mais pour sa mise en scène d'une commune apothéose aquatique des deux protagonistes noyés, lesquels deviennent, comme Glaucus, des divinités marines ${ }^{93}$. Bien qu'il n'y ait pas, dans ce cas, de métamorphose physique du

sont pas des inventions d'auteurs et gardent une connexion avec la tradition orale. Cf. également p. 124-126.

88. Sur ce romance cf. J. Caro Baroja, Algunos mitos españoles, p. 135-137 et 141-142 (n. 7). Un happy end artificiel et rationalisé conclut le pliego.

89. Cf. C. Gallini, op. cit., p. 65-66 et 73.

90. Cf. plusieurs récits de ce genre dans le traité du pseudo-Plutarque sur Les fleuves (C. Gallini, op. cit., p. 71-72).

91. C. Gallini, ibid., p. 76-78.

92. Mét., IV, 416-562. Ovide y revient dans les Fastes, VI, 473-562, à propos des Matralia.

93. Sur ce mythe et ses connexions proche-orientales cf. Corinne Bonnet, " Le culte de 
jeune garçon ni d'allusion à une quelconque hybridité, le fait que la divinisation de Mélicerte résulte de l'initiative de sa mère et passe par sa prise en charge par des dauphins (qui après son immersion se substituent à sa mère dans la conduite de son cursus initiatique) invite à une comparaison globale de ce mythe et de ses corrélats rituels avec certains éléments du cycle légendaire de Cola Pesce : j'y reviendrai ailleurs, notant seulement ici que, dans les deux cas, la mère est directement responsable du destin de son fils ${ }^{94}$, et qu'il existe une légende parahagiographique byzantine qui semble opérer la transition entre le mythe de Mélicerte et celui de Cola Pesce.

Il s'agit de la tradition médiévale relative à Nicolas le Pèlerin de Trani, ce berger grec du $\mathrm{XI}^{e}$ siècle qui avait été expulsé par sa mère exaspérée par son extravagante piété, puis jeté à la mer (pour la même raison) par les moines du monastère où il avait trouvé refuge (mais ensuite pris en charge et sauvé par des dauphins), enfin précipité par-dessus bord par les marins du bateau sur lequel il tentait de se rendre en Italie, mais encore sauvé, cette fois par une Dame venue du ciel, secours analogue à celui dont Ulysse naufragé bénéficia de la part d'Ino ${ }^{95}$. Finalement arrivé sur la côte des Pouilles, ce Nicolas partage donc le nom, quelques aventures marines, l'exclusion familiale et sociale et la patrie (d'adoption) du prodigieux nageur du détroit de Messine dont plusieurs versions anciennes précisent qu'il était d'origine apulienne. La dévotion originale de ce Mélicerte chrétien, ses bienfaits, ses accointances ichthyologiques et sa vocation pérégrinante se retrouvent dans l'abnégation gyrovague et serviable de Cola Pesce, auquel il a peut-être servi d'intermédiaire avec cet autre Nicolas, celui de Myra et de Bari, lui aussi venu d'Asie Mineure et installé post mortem en Apulie d'où il exerce sa sainte protection sur tout le monde de la chrétienté méditerranéenne.

À la différence de celle de Glaucus, donc, la métamorphose de Cola Pesce - qui pourtant lui ressemble fort - a quelque chose à voir avec sa mère, point important sur lequel il se rattache plutôt au modèle de Mélicerte et / ou de quelques autres personnages (comme les victimes de la Naïade de Nosala, également mentionnée par Ovide $)^{96}$ dont l'ichthyomorphose est imputable à une entité féminine ambivalente.

Cette constatation mamène à compléter mon exégèse mythique de ce personnage complexe et à confirmer l'hypothèse des composantes procheorientales de son dossier, développée dans mon antérieure investigation de

Leucothea et de Mélicerte, en Grèce, au Proche-Orient et en Italie ", Studi e Materiali di Storia delle Religioni, X (1), 52, 1986, p. 53-71, et pour les versions ovidiennes P. Perdrizet, op. cit., p. 207-217, et D. Mantzilas, op. cit., p. 180-181.

94. Dans une des versions orales recueillies par Pitrè (op. cit., p. 157) la mère de Cola "pi la pena, si mazzulió tantu, ca nni muriu "; même détail in G. Cavarra, op. cit., p. 130.

95. Cf. Gerardo Cioffari, San Nicola pellegrino, patrono di Trani, Bari, Centro Studi Nicolaiani, 1994, et Acta Sanctorum (2 juin, t. I, p. 245 sq).

96. Cf. supra n. 23. Cf. le passage de la Jérusalem délivrée mentionné supra n. 63, qui semble combiner le thème de Glaucus, celui de Circé et celui de cette naïade, dont les amants transformés en poissons par elle retrouveront forme humaine. 
ses rapports avec saint Nicolas. Je le ferai en soulignant la probable origine sémitique de ce corpus légendaire qui attribue à une malédiction maternelle l'immersion et la transformation en hybride marin infligées à notre héros comme à la plupart des riveraines espagnoles devenues sirènes.

Si l'invocation maternelle a une telle force contraignante c'est probablement, en deçà des réinterprétations démoniaques imaginées par les clercs, parce qu'elle procède d'une divinité. Une divinité féminine ambivalente qui n'est autre que la Dea Syria, à savoir Atargatis (alias Astarté), dont le culte s'est répandu dans tout le bassin méditerranéen dès l'époque hellénistique, plus anciennement encore dans les zones partiellement colonisées par les Phéniciens - entre autres GadesCadix et les côtes siciliennes ${ }^{97}$ - qui sont précisément celles où s'est enracinée le plus profondément la légende de Cola Pesce. La mythologie de cette déesse, liée à des rituels où interviennent le catapontisme et l'ichthyolâtrie, comporte des récits d'immersion et de transformation en poisson ${ }^{98}$. Dans ces mythes les incarnations de la divinité féminine sont en étroite association avec un jeune personnage masculin, qui est tantôt son fils, tantôt son amant (quand les deux ne sont pas coexistants, voire confondus), lequel partage le sort de celle dont il est le parèdre, au point de plonger avec elle et de subir la même ichthyomorphose.

Ovide n'ignorait rien de cette mythologie, comme en témoignent les allusions qu'il fait, dans les Métamorphoses et dans les Fastes, aux transformations et aventures aquatiques de "Dercetis la babylonienne ", à l'immersion de Vénus (alias Dione) et de Cupidon (alias Ichthys) dans l'Euphrate où des poissons les prennent en charge, en récompense de quoi ils feront l'objet d'une transformation en constellation, à l'histoire de la naïade de Nosala qui, avant de devenir elle-même poisson, infligeait à ses amants cette métamorphose ${ }^{99}$. Les légendes relatives à Sémiramis et à ses parèdres masculins, celles qui concernent Ino-Leucothea et Britomartis, autres plongeuses, relèvent du même complexe mythique $^{100}$, où le thème de la transgression (notamment sexuelle) et

97. Cf. C. Bonnet, Melqart. Cultes et mythes de l'Héraclès tyrien en Méditerranée (Studia Phoenicia VII), Presses de l'Univ. de Namur, 1988, et, pour le dossier ibérique, Antonio García y Bellido, "Deidades semitas en la España Antigua », Sefarad, 24, 1964, p. 12-40 et 237-275.

98. Cf. Joseph Fontenrose, "White Goddess and Syrian Goddess", in Semitic and Oriental Studies (Univ. of California Publ. in Semitic Philology), t. XI (= Mélanges W. Popper), Berkeley, 1951, p. 125-148, et, pour l'ensemble du dossier de la Déesse Syrienne et de ses sources, PaulLouis Van Berg, Corpus cultus Deae Syriae, 1. Les sources littéraires, vol. I, Répertoire des sources grecques et latines, vol. II, Étude critique des sources mythographiques grecques et latines, Leyde, Brill, 1972.

99. Cf. supra nn. 23 et 96, et P. Perdrizet, op. cit., p. 217-221. Sur les mentions ovidiennes relatives à Dercetis (alias la Vénus syrienne, alias Dione) cf. ibid., p. 193-197 et D. Mantzilas, op. cit., p. 591-593. Le sujet réapparaît dans les Fastes, II, 457-474 (à propos de la constellation des Poissons).

100. Cf. J. Fontenrose, op. cit.. Les noms des époux (Onnes, Ninus), puis du fils (Ninyas) de Semiramis (fille de Derceto) semblent bien renvoyer au monde ichthyologique : cf. W. Robertson Smith, "Ctesias and the Semiramis legend ", The English Historical Review, 2, 1887, p. 303-317 (p. 313-317). La même divinité est connue en Espagne, notamment à Gades, sous le nom de 
du châtiment intervient à plusieurs niveaux, servant en particulier à justifier et expliquer des processus initiatiques complexes et à rendre compte d'allusions rituelles devenues difficiles à déchiffrer.

Certes la mère de Cola Pesce ne se livre à aucune immersion, quoiqu'une version orale précise curieusement qu'elle se suicide (en plongeant à son tour ?) au moment où elle assiste à celle de son fils ${ }^{101}$. C'est cependant bien elle qui est à l'origine de cette mutation décisive. Une autre tradition locale sicilienne attribue même l'histoire entière de Cola à une sirène, qui périt comme lui au fond du détroit ${ }^{102}$. Je reviendrai ailleurs sur l'ensemble des (rares) autres connexions féminines du prodigieux nageur, notamment avec sa sœur, ses filles, la fille du roi, selon les quelques versions qui y font allusion, où transparaît parfois la fugitive mention d'une tragédie amoureuse, qui pourrait relever d'un croisement avec le cycle de ballades traditionnelles sur "la pesca del anillo", où certains ont voulu voir la racine de notre légende sicilienne ${ }^{103}$. Mais l'argument de ces chansons - qui portent sur la tentative, souvent fatale, risquée par un plongeur amoureux de récupérer l'anneau perdu (ou jeté) en mer, voire dans un puits, par une belle capricieuse -, lequel fait écho à la descente de Cola Pesce en quête de la coupe immergée dans le détroit par le roi, ne reconduitil pas lui aussi à un thème mythico-rituel lié au culte de la Dea Syria, dont les incarnations visibles sont précisément des poissons porteurs d'anneaux (attachés à leurs ouies) ${ }^{104}$ ?

Les remarques que je viens de formuler permettent d'envisager l'hypothèse selon laquelle les analogies relevées plus haut entre la métamorphose du Glaucus ovidien et celle de Cola Pesce ne pourraient s'expliquer totalement ni par un lien généalogique - au sens où le second serait une "survivance " locale du premier, ou le produit folklorisé d'une réminiscence littéraire - ni par une simple affinité typologique, mais reconduiraient à leurs dépendances respectives à l'égard d'un héritage (sémitique) commun.

"Venus Marina".

101. Cf. supra n. 94.

102. G. Pitrè, op. cit., p. 156-157.

103. Cf. G. B. Bronzini, La canzone epico-lirica nell'Italia centro-meridionale, Rome, A. Signerelli, 1956-1961, 2 vols., t. 2, p. 1-61, et Samuel G. Armistead et Joseph H. Silverman, En torno al romancero sefardi (Hispanismo y balcanismo de la tradición judeo-española), Madrid, Seminario Menéndez Pidal, 1982, p. 228-234 et 235-239. On sait que dans la majorité de ces chansons ("El buceador» ou "La pesca del anillo", etc.) l'issue la plus fréquente est la noyade du jeune plongeur. Dans plusieurs versions orales de la légende de Cola Pesce c'est bien un anneau qui, plutôt qu'une coupe, est jeté par le roi dans le détroit : l'épreuve est alors présentée comme ouvrant droit au mariage du vainqueur avec la princesse. Mais on sait que le roi ne tiendra pas sa promesse...

104. Cf. F. Delpech, « La sépulture subfluviale de Daniel et le mystère indo-européen du "Feu dans l'Eau" ", Voix des mythes, science des civilisations (Hommage à Philippe Walter), F. Vigneron et K. Watanabe (éds.), Berne: Peter Lang, 2012, p. 3-16. Je reviendrai ailleurs sur le cycle folklorique concernant les poissons qui avalent ou rapportent des anneaux immergés. 
Une origine babylonienne, médiatisée par la Crète, a pu être en effet envisagée avec vraisemblance pour les trois principaux Glaukos des traditions helléniques, dans les légendes desquels se retrouve l'association d'un catapontisme et d'un accès à une herbe d'immortalité, complexe thématique central dans la geste de Gilgamesh ${ }^{105}$, et j'ai moi-même supposé par ailleurs que les transformations en poissons du Messie marrane du Guadalquivir, du Lazarillo d'Anvers et de Cola Pesce avaient quelque lointaine parenté avec le mythe d'Oannes et le type babylonien de l'apkallu, sage ichthyomorphe faisant office de héros civilisateur antédiluvien ${ }^{106}$.

S'ily a donc finalement quelque ressemblance entrel'aventure métamorphique marine de Cola Pesce et quelques-unes de celles qui sont évoquées dans le poème ovidien il appert que, par un apparent paradoxe, l'analogie ne s'impose vraiment que dans les versions de sa légende qui ont été imaginées et ont principalement circulé dans un milieu illettré bien étranger à la tradition littéraire latine, mais peut-être plus proche des sources traditionnelles d'Ovide qu'il ne l'était lui-même. Ceux qui connaissaient Ovide n'ont par contre guère pu envisager le rapprochement, car à leurs yeux Cola Pesce, dont ils connaissaient manifestement l'existence par la vox populi, était principalement, ne pouvait être, qu'un nageur exceptionnel et fameux du temps jadis sur lequel on racontait des histoires plus ou moins invraisemblables : pour eux la métamorphose n'était plus qu'une fiction poétique relevant du merveilleux ou une allégorie morale, et ni les humains ni les tritons et les sirènes, dont on continuait à affirmer l'existence dans les cosmographies, lesquels étaient considérés comme des êtres naturels, ne pouvaient y être sujets. Si le nageur sicilien, devenu " menzu pisci e menzu оти " suite à une mystérieuse transmutation, des récits de pêcheurs et de marins est comparable aux héros ovidiens, ce ne peut être que par un processus de remythisation - combinant peut-être survivances, résurgences et réinventions - de la part de gens de mer incultes mais croyant encore à ce à quoi Ovide lui-même ne croyait probablement déjà plus...

105. Cf. B. Deforge, op. cit., et I. Palladino, op. cit.

106. F. Delpech, «De saint Nicolas à Cola Pesce " (cit. supra n. 5) n. 75, id., "Lazare, l'eau, le vin et les thons" (cit. supra n. 9), p. 323, n. 52, et p. 325, et id., "Du folklore au discours prophétique...» (cit. supra n. 38), p. 402. 
